\title{
One-component nanocomposites based on polymer-grafted cellulose nanocrystals
}

Sandra Wohlhauser, Tobias Kuhnt ${ }^{\S}$, Worarin Meesorn, Lucas Montero de Espinosa, Justin O. Zoppe, Christoph Weder*

Adolphe Merkle Institute, University of Fribourg, Chemin des Verdiers 4, 1700 Fribourg, Switzerland

$\S$ Department of Complex Tissue Regeneration, MERLN Institute for Technology Inspired Regenerative Medicine, Maastricht University, 6229 ER, Maastricht, the Netherlands

ABSTRACT. Cellulose nanocrystals (CNCs) are widely used as reinforcing filler in polymers, due to their exceptionally high stiffness and strength and because the biological species from which they are isolated represent renewable resources. However, aggregation of the CNCs, which is concomitant with limited reinforcement, is often difficult to avoid. One-component nanocomposites (OCNs) based on polymer-grafted nanoparticles can solve this problem, because this approach affords, by design, materials in which no such aggregation is possible. At the same time, chain entanglements between the CNC-grafted polymer chains provide stress-transfer among the particles. To demonstrate this, we investigated OCNs based on polymethacrylate-grafted CNCs. A previously un-accessed compositional space, i.e., OCNs with a CNC content of 10 or $20 \mathrm{wt} \%$, was explored. Cotton linter-based CNCs were modified via surface-photoinitiated free radical polymerization, which involved the functionalization of the CNC surfaces with benzophenone moieties as photo-radical initiator species, and the subsequent surface-photoinitiated polymerization of methyl or hexyl methacrylate under UVirradiation at $365 \mathrm{~nm}$. The resulting particles readily dispersed in THF. Solvent-casting and compression-molding afforded films of homogeneous appearance, which display remarkable 
improvements in stiffness or toughness and strength in comparison to conventional twocomponent nanocomposites of unmodified CNCs and the respective polymers.

\section{INTRODUCTION}

In order to enhance their dispersibility in solvents or solid (polymer) matrices, the surface of nanoparticles (NPs) is often decorated with compatibilizing molecules. ${ }^{1}$ This approach has been applied to many different matrix/nanoparticle combinations. ${ }^{2,3}$ In addition to physisorption of small molecular surfactants or amphiphilic (co)polymers on the NPs surface, polymers can be covalently grafted to or from the surface of the NPs to compatibilize them with a given polymer, but especially at high NP loading, phase separation or de-mixing remain an omnipresent obstacle. ${ }^{4}$ This problem can be solved by creating so-called one-component nanocomposites (OCNs), i.e., materials resulting from the assembly of polymer-grafted or "hairy" NPs (HNPs) in the absence of any additional polymer matrix. ${ }^{5}$ HNPs consist of a central core that is surrounded by a polymer corona. Unlike conventional polymer nanocomposites that consist of at least two separate components, OCNs can, by design, not suffer from mixing and phase separation problems, because their components are covalently linked, and if the grafting is homogeneous, aggregation of CNCs should also be suppressed. Strictly speaking, this design feature does not qualify these materials as composites in the classical sense, but nevertheless the term "one-component nanocomposites" is used as it concisely describes their structure. ${ }^{5}$ OCNs can be easily processed via either solvent casting of the HNP dispersion or by melt processing the dried material at a temperature above the glass transition or the melting point of the grafted polymer. OCNs based on hairy spherical NPs have been shown to display anisotropic elastic properties, as a result of substrate-induced formation of layered assemblies. ${ }^{6}$ Previous works in the field of hairy nanoparticles have been mainly focused on spherical NPs, ${ }^{5}$ such as silica, albeit the use of high-aspect-ratio nanoparticles can 
significantly enhance the mechanical properties of the final material. In this context, we surmised that OCNs based on polymer-grafted cellulose nanocrystals (CNCs) would be an interesting materials family to investigate. To the best of our knowledge, only two experimental studies ${ }^{7,8}$ and two computational simulations, ${ }^{9,}{ }^{10}$ have been published on OCNs based on hairy CNCs.

CNCs are rod-like NPs that are readily isolated through selective hydrolysis from renewable bio-sources, such as higher plants, bacteria, and tunicates, among others. ${ }^{11,12} \mathrm{CNCs}$ consist of linear homopolysaccharides composed of anhydroglucopyranose repeat units that are connected via $\beta$ (1-4') glycosidic bonds. ${ }^{11} \mathrm{CNCs}$ are moderately to highly crystalline (54-88\%) with an aspect ratio (10-170) that depends mainly on the raw material, but also on the hydrolysis conditions. ${ }^{12} \mathrm{CNCs}$ are frequently used as reinforcing filler in polymer nanocomposites, due to their low density, high tensile strength $(7.5-7.7 \mathrm{GPa})^{13}$ and stiffness $(57-151 \mathrm{GPa}),{ }^{12}$ and the renewable nature of the sources from which they are isolated. ${ }^{14}$ Data also suggest that unlike other nanofillers, CNCs have a limited associated toxic potential when investigated under realistic exposure scenarios and doses. ${ }^{15}$

The use of CNCs as reinforcement agent in a polymeric matrix was pioneered by Favier et al., who observed an improvement of the mechanical properties of poly(styrene-co-butyl acrylate) when a latex of this polymer was combined with CNCs extracted from tunicates and processed into films. ${ }^{16,17}$ Since then, CNC-polymer nanocomposites have been widely investigated and their properties are now well-understood. ${ }^{14}$ The mechanical reinforcement in polymer/CNC nanocomposites is generally accepted to take place through a hydrogen-bonded $\mathrm{CNC}$ network that percolates within the polymer matrix, which is responsible for efficient stress transfer. ${ }^{17}$ The reinforcement achieved is typically well predicted by a percolation model, which considers that the $\mathrm{CNC}$ percolating network is formed over a certain filler content (i.e., 
percolation threshold). ${ }^{18,19}$ However, in many cases the actual reinforcement does not match with the prediction of this model. ${ }^{20,21}$ One possible reason is that it does not take into account $\mathrm{CNC}$ aggregation, which we have recently shown to be important. ${ }^{22}$ The remaining challenge is thus to enhance the dispersion of CNCs within the polymer matrix. Many efforts have been directed to address this problem by increasing the hydrophobicity of the CNCs via surface modification. ${ }^{23}$ For instance, stable dispersions of CNCs/poly(lactic acid) in chloroform ${ }^{24}$ or $\mathrm{CNCs} /$ atactic poly(propylene) in toluene ${ }^{25}$ have been reported after physically coating the $\mathrm{CNC}$ surface with surfactants. The physical adsorption of polymers on CNCs was also shown to improve their dispersibility. ${ }^{26}$ For example, after adsorbing poly(ethylene oxide), the CNCs dispersed more readily in (hydrophobic) low-density polyethylene and the resulting nanocomposites could be processed via melt-extrusion into objects of homogeneous appearance.

Another strategy to enhance the dispersibility of CNCs is to chemically modify their surface with motifs that are similar to the polymer matrix or sites that display specific interactions with the matrix. ${ }^{23}$ Examples include the TEMPO-mediated oxidation of the surface $\mathrm{OH}$-groups, ${ }^{27}$ the attachment of small molecules such as organic acid chlorides, ${ }^{28}$ or acetic anhydride, ${ }^{29}$ and the introduction of long polymer chains. ${ }^{30,31}$

As discussed above, one-component nanocomposites (OCNs) based on polymer-grafted nanoparticles promise to be another solution to the dispersion problem, because their architecture prevents macrophase separation. At the same time, chain entanglements between the CNC-grafted polymer chains can enable stress-transfer among the particles. Indeed, the polymer chain length, the grafting density and the polymer structure also should influence the mechanical properties of CNC-based OCNs according to the recent computational predictions reported by Hansoge et al. ${ }^{9,} 10$ 
Polymer-grafted CNCs can be synthesized via "grafting-from", "grafting-to", or "graftingthrough" approaches involving functional groups on the CNCs surface. ${ }^{3,} 32$ The grafting-from approach, which was utilized here, involves the functionalization of the CNCs with polymer brushes by way of surface-initiated polymerization from initiator groups immobilized on the NPs' surface. This framework generally leads to polymer grafts with a well-controlled length and high polymer grafting density. ${ }^{3}$ The first example of OCNs based on polymer-grafted CNCs, using a grafting-from approach, was reported by Chen et al., ${ }^{7}$ who functionalized CNCs with semicrystalline poly( $\varepsilon$-caprolactone) via surface-initiated ring-opening polymerization. OCNs with a CNC content of between 4 and $8 \mathrm{wt} \%$ proved to be melt-processable and the authors reported a non-linear dependence between the $\mathrm{CNC}$ content and the OCN mechanical properties. Unfortunately, no comparison with conventional composites was made and no correlation between the PCL graft structure and the properties of the OCNs could be established. In another study, Chang et al. reported the synthesis of CNCs-g-poly(ethynylenefluorene) through Sonogashira coupling via a grafting-from approach, ${ }^{8}$ but the mechanical properties of the material were not investigated.

Here, we report the synthesis of amorphous, polymethacrylate-grafted CNCs through a synthetically undemanding free radical polymerization protocol. It involves the surface functionalization of CNCs with a benzophenone derivative that serves as radical photoinitiator for the surface-initiated photopolymerization of methyl and hexyl methacrylate upon UVirradiation. The morphology and thermomechanical properties of the OCNs made from these "hairy" CNCs were investigated and the materials were found to display remarkable improvements in stiffness or toughness and strength in comparison to conventional twocomponent reference nanocomposites consisting of unmodified $\mathrm{CNCs}$ and the respective polymethacrylate. 


\section{RESULTS AND DISCUSSION}

The synthesis of polymethacrylate HNPs (PMA-HNPs) via surface-initiated free radical photopolymerization from the surface of CNCs was performed using CNCs whose surfaces were modified with benzophenone (BP) moieties as photoactive radical initiator species (Scheme 1). CNCs were extracted via sulfuric acid hydrolysis from cotton according to a wellestablished protocol. ${ }^{20}$ The CNCs thus produced had an average length of $117 \pm 28 \mathrm{~nm}$ and an average width of $15 \pm 2 \mathrm{~nm}$ (established by image analysis of transmission electron microscopy images, Figure S1) and a surface charge density of $115 \mathrm{mmol} / \mathrm{kg} \mathrm{R}-\mathrm{OSO}_{3} \mathrm{H}$ and $21 \mathrm{mmol} / \mathrm{kg}$ $\mathrm{COOH}$ groups (established by conductometric titration of unmodified CNCs with $0.01 \mathrm{M}$ $\mathrm{NaOH}$ solution, Figure S2), which are introduced during hydrolysis. ${ }^{33}$ Applying conditions reported by Biyani et al., ${ }^{34}$ the CNCs were dispersed in water-free DMF and reacted with 4benzoylphenyl-(6-isocyanatohexyl)carbamate (1, Figures S3 and S4) in order to functionalize the CNCs surface with BP moieties (Scheme 1). After purification by repeated ultracentrifugation and washing with DMF and THF, the resulting CNC-g-BPs were dried in vacuum and kept in an amber glass vial to protect them from light. FTIR spectra show the characteristic signals of urethane bonds at 1615 and $1576 \mathrm{~cm}^{-1}$, confirming the successful attachment of the BP-containing radical initiation sites (Figure 1). The absence of the absorption band at $2265 \mathrm{~cm}^{-1}$, characteristic of the isocyanate group, further reveals the complete removal of unreacted compound 1. An initiator grafting density $\left(\sigma_{i}\right)$ of $0.8 \mathrm{BP}$ molecules $/ \mathrm{nm}^{2}$ was established by UV-vis spectroscopy following the methodology reported by Biyani et al. ${ }^{34}$ For this purpose, a calibration curve was created using BP compound $\mathbf{1}$ (Figures S5 and S6), which was employed to determine the BP content of a CNC-g-BP dispersion. All samples were analyzed by comparing the absorbance at $285 \mathrm{~nm}$ and the absorbance of $100 \mu \mathrm{g} / \mathrm{mL}$ dispersions of CNC-g-BP was corrected by subtracting the signal of corresponding dispersions of unmodified CNCs in order to account for scattering effects. 


\section{CNCs}
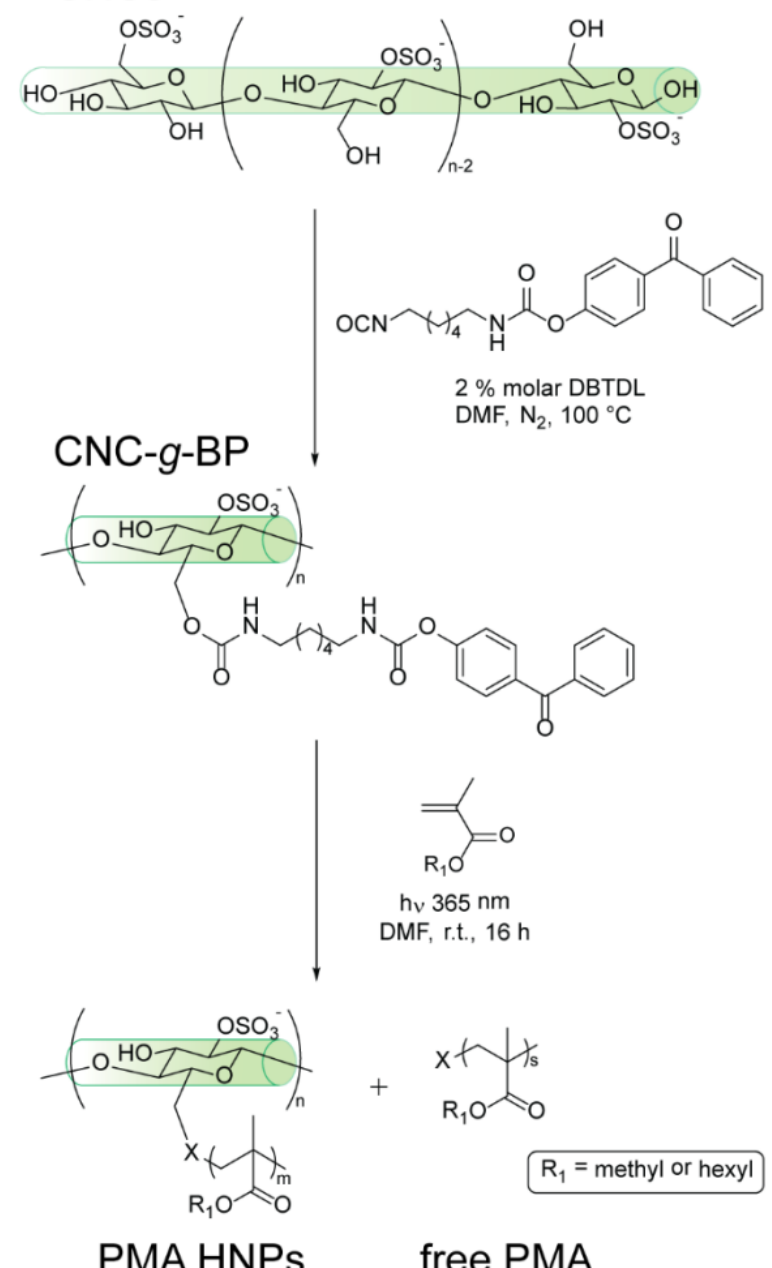

PMA HNPs free PMA

Scheme 1. Synthesis of polymethacrylate HNPs via the surface functionalization of CNCs with 4-benzoylphenyl-(6-isocyanatohexyl)carbamate (1) and photoinitiated grafting of polymethacrylates from the surface of modified $\mathrm{CNCs}(\mathrm{CNC}-g$-BP). Due to the initiation mechanism (see text), free PMA is also produced.

As a next step, MMA or HMA were polymerized from the surface of CNC-g-BP via surfacephotoinitiated FRP (Scheme 1). MMA and HMA were chosen as monomers in order to investigate the properties of CNC-based OCNs with glass transition temperatures $\left(T_{g}\right)$ above and below room temperature. As a Norrish Type II photoinitiator, benzophenone undergoes 
intersystem crossing to a reactive triplet excited state upon irradiation at 350-360 nm, which in turn causes the carbonyl group to abstract any nearby aliphatic hydrogen (i.e., from the surface of the CNCs, the solvent, or the monomer), yielding ketyl and carbon-centered radicals. ${ }^{35}$ The initiation process can thus occur from radicals on the surface of the CNCs or from monomers/solvent radicals in solution, which affords free (i.e., not CNC-bound) polymer chains. At least for research purposes, this is an attractive feature as it affords, simultaneous to the growth of polymer-grafts from the CNCs, free macromolecules in solution, which can be easily isolated and characterized. As ungrafting polymers from $\mathrm{CNCs}$ is not straightforward, it has become common ${ }^{36,37}$ to rely on the characterization of such free polymer populations instead of the grafted polymer ${ }^{38,39}$, but in most surface-initiated polymerizations, a sacrificial initiator (i.e., one that is not attached to the nanoparticles) must be added to the reaction mixture.

Thus, MMA and HMA were polymerized in the presence of CNC-g-BP in anhydrous DMF using two 6 Watt UV lamps emitting at $365 \mathrm{~nm}$ (Scheme 1). The reaction time was fixed to 16 $\mathrm{h}$ and the monomer concentration was varied to control the amount of polymer grafted onto the CNCs. The HNPs were then separated by several cycles of ultracentrifugation and washing with THF. The free polymers were isolated from the supernatant by concentration and precipitation in cold methanol (Table 1). The ${ }^{1} \mathrm{H}$ NMR spectra of the free polymers produced during the synthesis of PMMA- and PHMA-HNPs match those of PMMA and PHMA standards, respectively (Figure S7). For reference purposes, free PMMA (number-average molecular weight, $M_{n}=161 \mathrm{kDa}$, dispersity, $\left.\oslash=M_{w} / M_{n}=1.8\right)$ and PHMA chains $\left(M_{n}=125\right.$ $\mathrm{kDa}, Ð=2.3$ ) isolated after the synthesis of PMMA-HNP-10\% and PHMA-HNP-10\%, respectively, were used in this study for the fabrication of two-component nanocomposites containing equivalent weight percentage of CNCs than the synthesized HNPs, i.e., 10 and $20 \mathrm{wt} \%$. 
The monomer conversion into grafts was determined based on the total amount of monomer present in the reaction mixture, and the weight of the polymer grafts produced, which in turn was determined from the mass difference between the isolated HNPs and the initial amount of CNC-g-BP (Table 1). The monomer conversion into grafts varied between 15 and 20\%, which may appear low, but due to the specific initiation mechanism (vide supra) a substantial amount of free polymer was produced in solution (28-60\%). HNPs in which the CNC weight fraction was ca. $10 \mathrm{wt} \%$ (referred to as PMMA-HNP-10\% and PHMA-HNP-10\%) were obtained when the monomer concentration in the feed was 4.7 $\mathrm{M}$ and 2.5 $\mathrm{M}$ for MMA and HMA, respectively, and the CNC content was doubled to $20 \mathrm{wt} \%$ (PMMA-HNP-20\% and PHMA-HNP-20\%) when the monomer concentrations were cut in half (i.e., $2.34 \mathrm{M}$ and 1.27 M for MMA and HMA). A further decrease of the HMA concentration to 0.6 and $0.3 \mathrm{M}$ yielded HNPs with a CNC content of up to $58 \mathrm{wt} \%$, but we note that the reproducibility of reactions with low monomer concentrations was, in contrast to the above reactions (Table 1) not very good (Table S1).

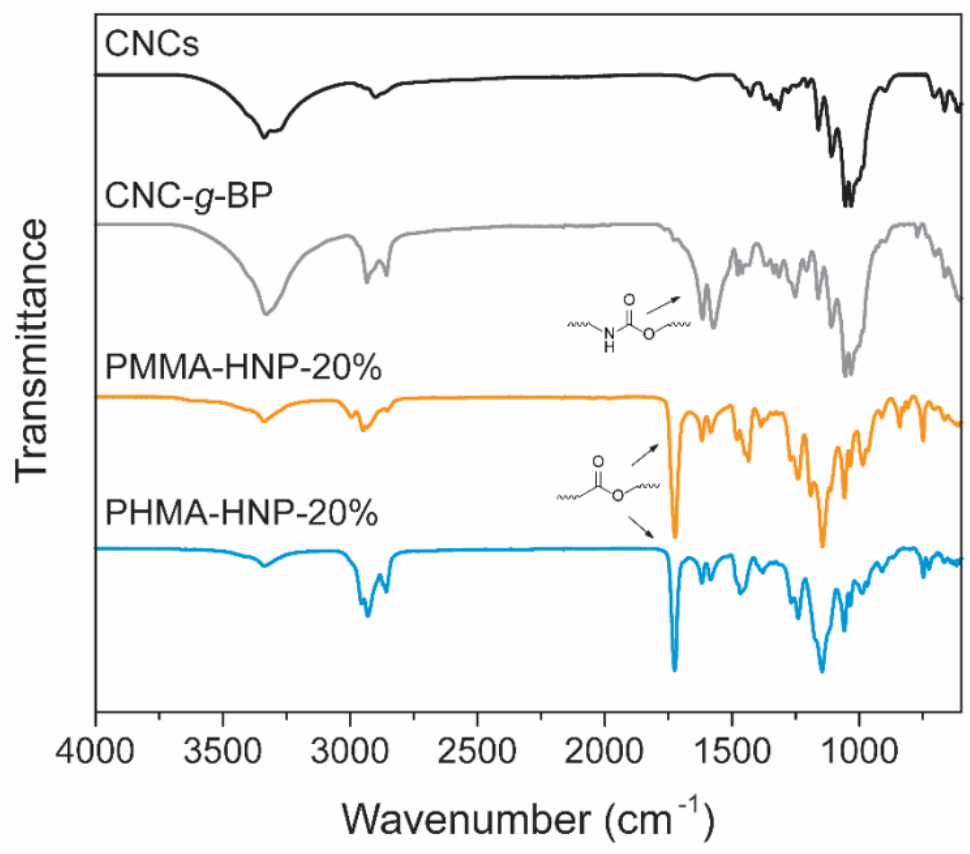

Figure 1. FTIR spectra of unmodified CNCs, CNC-g-BP, PMMA- and PHMA-based HNPs containing $20 \mathrm{wt} \% \mathrm{CNCs}$ (top to bottom). 
The presence of PMMA and PHMA grafts in the HNPs is evident from the FTIR spectra, which show intense carbonyl ester peaks at 1726 and $1723 \mathrm{~cm}^{-1}$, respectively (Figure 1 and Figure S8). Furthermore, the increased intensity of alkyl group peaks in the range of 2954$2858 \mathrm{~cm}^{-1}$ in the FTIR spectra of the PHMA-HNPs is indicative of the hexyl group of the PHMA repeating unit. ${ }^{1} \mathrm{H}$ NMR spectra of well-dispersed PMMA and PHMA HNPs in $\mathrm{CDCl}_{3}$ also reflect the polymerization of MMA and HMA from the surface of CNCs by the presence of broad signals that are in good agreement with the expected structures (Figure S9). The formation of PMMA and PHMA grafts and the gravimetrically determined compositions were finally confirmed by elemental analysis data (Table S2), which show a good match between the experimentally determined and theoretical compositions based on the molecular constitution of the repeating units of $\mathrm{CNC}-g$-BP and the grafted polymer and considering the gravimetrically determined graft fractions. In the light of typical variations seen for the elemental analyses of modified $\mathrm{CNCs},{ }^{40}$ the experimentally determined compositions of HNPs show a good agreement with the elemental composition of the material. In the case of CNC-g$\mathrm{BP}$, the experimentally determined carbon content is higher (48.9\% vs. $46.6 \%)$ than the one determined theoretically, which may be an indication that the content of BP molecules determined via UV-vis spectroscopy could has been underestimated.

In order to cleave (a portion of) the polymers from the CNCs and allow for their molecular characterization in solution, chlorosilane-induced cleavage reactions of the urethane bonds were conducted under mild conditions in the presence of tertiary amines (Scheme 2). ${ }^{41}$ Only a fraction of polymethacrylate chains could be degrafted through this approach (around $30 \mathrm{wt} \%$ of the total grafts in the case of PMMA-HNPs and up to $56 \mathrm{wt} \%$ in the case of PHMA-HNPs, Table S3), which suggests that a significant fraction of the grafted chains are linked to the CNCs surface through C-C bonds. These results are consistent with the initiation process in 
which the polymerization can start from any carbon-centered radical due to the nature of benzophenone photoinitiator species (vide supra).

Table 1. Summary of the results of surface photoinitiated FRP of MMA and HMA from CNC$g$-BP, including HNP compositions, monomer conversions, number-average molecular weight $\left(M_{n}\right)$ and dispersity $(Ð)$ of surface-grafted and free polymers, polymer grafting densities ( $\left.\sigma_{\text {grafts }}\right)$ and initiator efficiencies $\left(\sigma_{\text {grafts }} / \sigma_{\mathrm{i}}\right)$. Data are averages of three separate reactions per composition, but molecular weight data are quoted for the individual samples.

\begin{tabular}{|c|c|c|c|c|c|c|c|c|c|c|}
\hline \multirow[b]{2}{*}{ Sample } & \multicolumn{2}{|c|}{ HNP composition } & \multicolumn{5}{|c|}{ Surface-grafted polymer } & \multicolumn{3}{|c|}{$\begin{array}{c}\text { Free polymer in } \\
\text { solution }\end{array}$} \\
\hline & 递 & 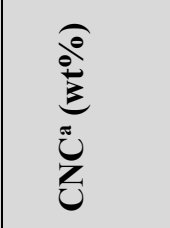 & 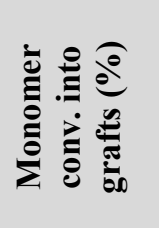 & 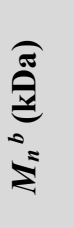 & İ & 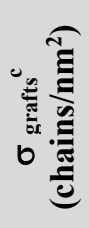 & $\begin{array}{l}I \\
I \\
\overline{0} \\
\frac{1}{2} \\
\frac{2}{50} \\
0 \\
0\end{array}$ & 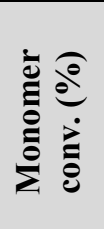 & 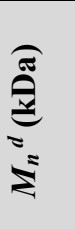 & $\begin{array}{l}I \\
\stackrel{1}{a}\end{array}$ \\
\hline \multirow{3}{*}{ PMMA-HNP-10\% } & \multirow{3}{*}{$92 \pm 1$} & \multirow{3}{*}{$8 \pm 1$} & \multirow{3}{*}{$20 \pm 1$} & & & & & \multirow{3}{*}{$30 \pm 2$} & 161 & 1.8 \\
\hline & & & & & & & & & 148 & 1.7 \\
\hline & & & & 271 & 2.2 & 0.15 & 0.18 & & 166 & 1.8 \\
\hline \multirow{3}{*}{ PMMA-HNP-20\% } & \multirow{3}{*}{$82 \pm 1$} & \multirow{3}{*}{$18 \pm 1$} & \multirow{3}{*}{$15 \pm 1$} & & & & & \multirow{3}{*}{$28 \pm 1$} & 77 & 1.9 \\
\hline & & & & 163 & 2.5 & 0.10 & & & 82 & 18 \\
\hline & & & & 133 & 2.6 & 0.12 & 0.15 & & 73 & 1.7 \\
\hline \multirow{3}{*}{ PHMA-HNP-10\% } & \multirow{3}{*}{$90 \pm 2$} & \multirow{3}{*}{$10 \pm 2$} & \multirow{3}{*}{$16 \pm 4$} & & & & & \multirow{3}{*}{$60 \pm 5$} & 125 & 2.3 \\
\hline & & & & 232 & 2.0 & 0.12 & 0.15 & & 100 & 24 \\
\hline & & & & 216 & 2.2 & 0.13 & 0.16 & & 105 & 1.7 \\
\hline \multirow{3}{*}{ PHMA-HNP-20\% } & \multirow{3}{*}{$80.7 \pm 0.3$} & \multirow{3}{*}{$19.3 \pm 0.3$} & \multirow{3}{*}{$14.6 \pm 0.3$} & & & & & \multirow{3}{*}{$32 \pm 8$} & 67 & 1.9 \\
\hline & & & & 371 & 1.9 & 0.04 & 0.05 & & 60 & 1.6 \\
\hline & & & & 391 & 2.1 & 0.04 & 0.05 & & 67 & 1.9 \\
\hline
\end{tabular}

a Determined gravimetrically by weighing the CNCs before and after the polymerization. bDetermined by SEC of partially degrafted polymers and calibration with PMMA standards. 'Determined from gravimetrical analysis considering an average specific surface of $174 \mathrm{~m}^{2}$ (see Supporting Information). ${ }^{\mathrm{d}}$ Determined by SEC of isolated free polymer chains in solution and calibration with PMMA standards. 

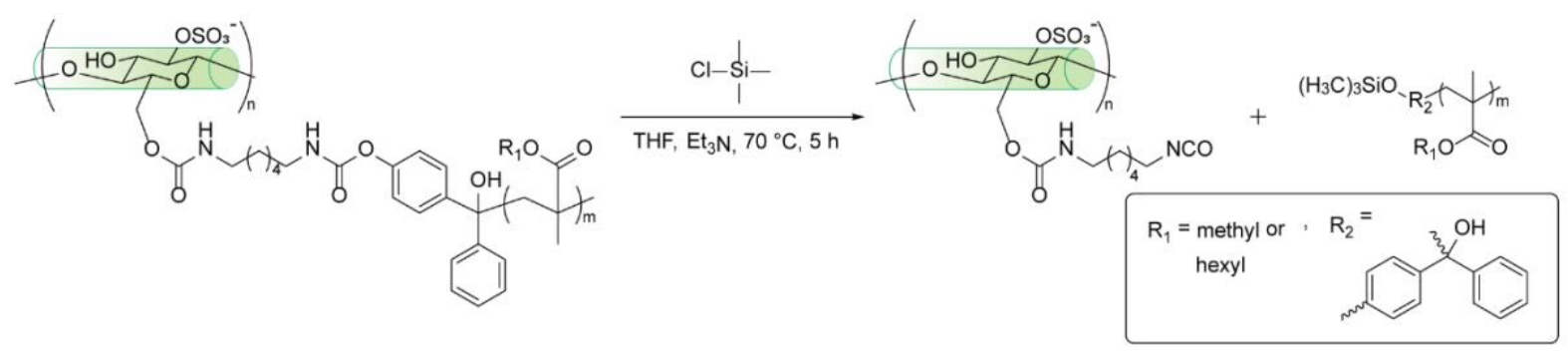

Scheme 2. Chlorosilane-induced cleavage reaction of urethanes bond from the surface of PMA HNPs in order to cleave the grafted PMA chains.

The ${ }^{1} \mathrm{H}$ NMR spectra of PMMA and PHMA degrafted from polymethacrylate HNPs containing $10 \mathrm{wt} \%$ of CNCs (Figures 2a and S10a, respectively) are similar to those of the reference materials obtained from the free polymer chains (Figure S7) isolated after the synthesis of HNPs and reveal the characteristic signals of these polymers. The SEC traces of PMMA and PHMA degrafted from PMMA- and PHMA-HNPs containing 10 and $20 \mathrm{wt} \%$ of CNCs are shown in Figures $\mathbf{2} \mathbf{b}$ and $\mathbf{S 1 0 b}$ (solid lines), and the corresponding $M_{n}$ and $Ð$ are summarized in Table 1 and Table S3. In all cases, the dispersity corresponds to polymers grown via free radical polymerization (1.9-2.6). The $M_{n}$ values of PMMA degrafted from PMMA-HNP-10\% and PMMA-HNP-20\% are around 266/271 and 133/163 kDa, respectively. The data are quoted for two different reactions per compositions and the similarity reflects that the process used is very reproducible. The difference of the molecular weights between the compositions is consistent with the much higher initial monomer concentration in the reaction feed for PMMA-HNP-10\%. Both HNPs feature grafts of a much higher $M_{n}$ than the free polymer chains isolated from the same reaction mixture, which appears to indicate that termination reactions are suppressed when the growing chains are tethered to the CNCs and display a lower translational mobility than free propagating chains. Based on the weight fraction (determined gravimetrically) and the $M_{n}$ of grafts, a PMMA grafting density of 0.15 
and 0.10/0.12 chains $/ \mathrm{nm}^{2}$ was estimated for PMMA-HNPs containing 10 and $20 \mathrm{wt} \%$ of CNCs, respectively, using the methodology proposed by Majoinen et al. (see Supporting Information ). ${ }^{36}$ The $M_{n}$ values of PHMA degrafted from PHMA-HNP-10\% and PHMA-HNP$20 \%$ are $216 / 232$ and $371 / 391 \mathrm{kDa}$, respectively, resulting grafting densities of $0.13 / 0.12$ and 0.04 chains $/ \mathrm{nm}^{2}$, respectively. In the case of the PHMA-HNPs, DMF is a poor solvent for the grafted chains in comparison with PMMA chains due to their larger aliphatic side chains making them less polar. As a result, the PHMA chains become more packed at a determined polymer concentration, which makes the PHMA chains inaccessible to monomer, as the propagating chains become buried by the compacted coils. This behavior explains the low monomer conversion into grafts.
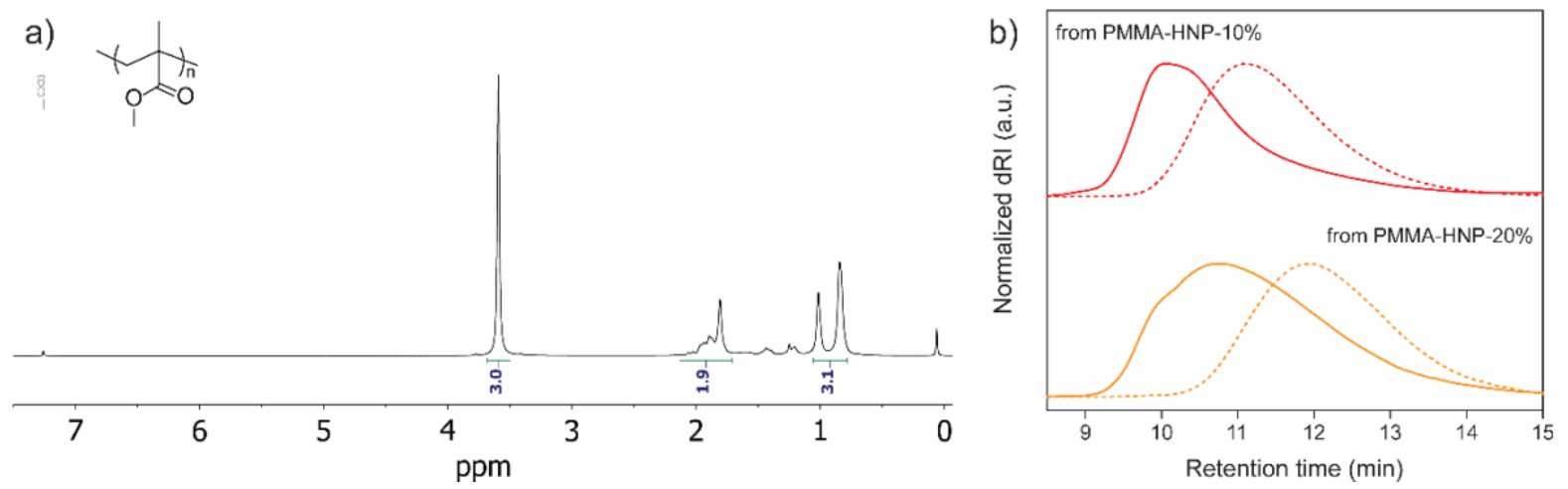

Figure 2. (a) ${ }^{1} \mathrm{H}$ NMR spectrum of PMMA degrafted from PMMA-HNP-10\% in $\mathrm{CDCl}_{3}$. (b) SEC traces of degrafted (solid line) and free (dashed line) PMMA isolated from PMMA-HNP10\% (top) and PMMA-HNP-20\% (bottom) using THF as eluent.

A comparison of the SEC data of degrafted and free PHMA polymers (Figures $\mathbf{2 b}$ and $\mathbf{S 1 0 b}$, Table 1) shows that the molecular weight of the free polymers is lower than tethered polymer chains. Other authors have also reported longer chains for the grafts than for free polymers, ${ }^{39}$, 42 and as above mentioned the effect appears to be a consequence of the reduced mobility of the tethered propagating chains, which in turn limits chain termination. The slight broadening of the molecular weight distributions observed for the PMMA synthesized via surface-initiated 
polymerization is in good agreement with the behavior predicted by Turgman-Cohen and Genzer, i.e. that the dispersity of polymers grown from flat surfaces is larger than that of polymers in bulk, and that it is dependent of the initiator grafting density, i.e., the dispersity increases with grafting density. ${ }^{43,44}$

As mentioned above, the HNPs were made from CNCs extracted via sulfuric acid hydrolysis and this process introduces sulfate half-ester groups $(115 \mathrm{mmol} / \mathrm{kg})$ on the CNCs surface. Considering the known fraction of cellulose chains on the CNCs surface (i.e., 0.19), ${ }^{45}$ one can calculate that about $7 \%$ of the reactive surface $\mathrm{OH}$ groups were substituted by sulfate half-ester groups during the hydrolysis, which translates into a slight reduction of the initiator grafting density. Based on a study of Tisuji et al., ${ }^{46}$ one can speculate that the presence of these negatively charged sulfate ester groups on the surface of CNCs promotes the migration of free radicals across the $\mathrm{CNCs}$ surface by sequential chain transfer reactions, thus enhancing chain recombination. This is consistent with a tail at high elution times in the SEC traces of degrafted polymethacrylates (Figures $\mathbf{2 b}$ and $\mathbf{S 1 0 b}$ ). On the other hand, such tail is absent in the SEC traces of free polymers, which supports the conclusion that chain recombination is more apt to happen on the CNCs surface on account of a higher local concentration of radicals than in the solution. 

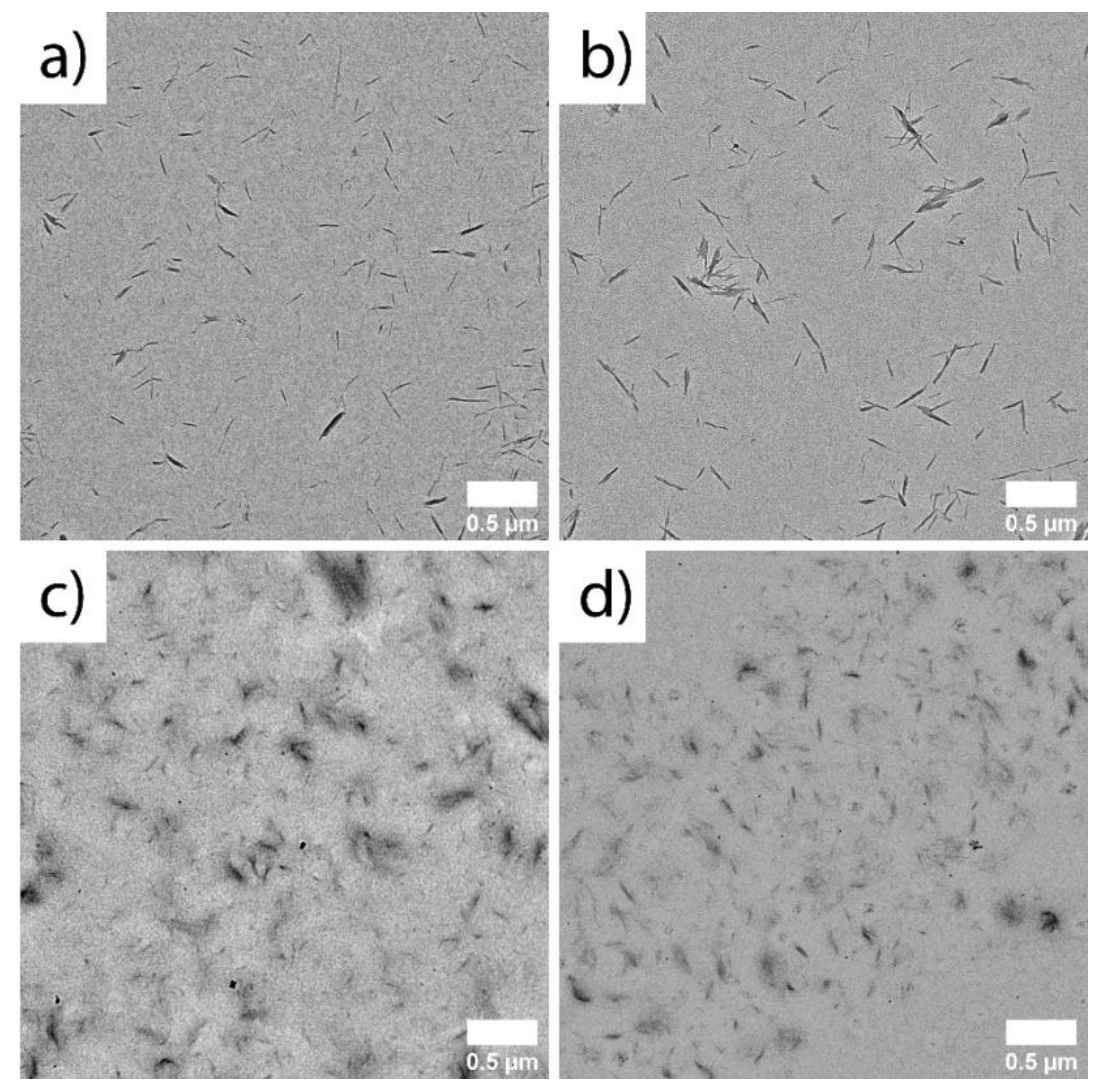

Figure 3. TEM micrographs of (a) unmodified CNCs, (b) CNC-g-BP, and polymethacrylate HNPs from dispersed (c) PHMA-HNP-10\% and (d) PMMA-HNP-10\%, scale bar $=500 \mathrm{~nm}$.

To explore how the surface modification affected the polarity and morphology of the CNCs, the various particle types were dispersed in a range of solvents, deposited onto transmission electron microscopy (TEM) grids, and imaged by TEM (Figure 3). The unmodified CNCs, which readily dispersed in deionized water, had a rod-like shape with a length of $117 \pm 28 \mathrm{~nm}$ and width of $15 \pm 2 \mathrm{~nm}$ according to image analysis (Figure 3a). The CNC-g-BP, which dispersed well in DMF, displayed similar morphology and dimensions (length $=113 \pm 33 \mathrm{~nm}$, width $=11 \pm 2 \mathrm{~nm}$ ) as unmodified CNCs (Figure 3b). The CNC-based HNPs could easily be dispersed in chloroform or THF, but not in water or DMF. The TEM micrographs of these HNPs show individual rod-like cores that are surrounded by a low-contrast corona, which was ascribed to the amorphous polymethacrylate grafts (Figure 3c-d). The HNPs seem to have 
shorter CNCs cores, but this effect is ascribed to the fact that they do not aggregate, in contrast to the neat $\mathrm{CNCs}$ or the $\mathrm{CNC}-\mathrm{g}$-BP.

The CNC-based HNPs were processed into OCN films with a thickness of ca. $0.2 \mathrm{~mm}$ by compression molding at $160{ }^{\circ} \mathrm{C}$ (PMMA-HNPs) and $130{ }^{\circ} \mathrm{C}$ (PHMA-HNPs). For reference purposes, we also produced conventional two-component composites containing 10 and 20 wt $\%$ of CNCs by first solution-casting from THF a dispersion of unmodified CNCs and free reference polymethacrylates (isolated from the synthesis of PMMA-HNP-10\% and PHMA$\mathrm{HNP}-10 \%$ ), vacuum-drying and finally compression molding at $130{ }^{\circ} \mathrm{C}$ (PMMA/CNCs) and $100{ }^{\circ} \mathrm{C}$ (PHMA/CNCs). Figure 4 shows photographs of the resulting OCN films, which clearly reveal a high level of transparency and homogeneity. This behavior contrasts that of the twocomponent reference composites, in which CNC aggregation is at play (Figure S11), and is a first indication of the excellent dispersion of the CNCs in the OCNs, which is imparted by the molecular connectivity of the building blocks. Figure 4 also qualitatively reflects the high flexibility of the PHMA-OCNs.

\section{PMMA-OCN10 PMMA-OCN10

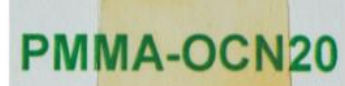 \\ PMMA-OCN20}
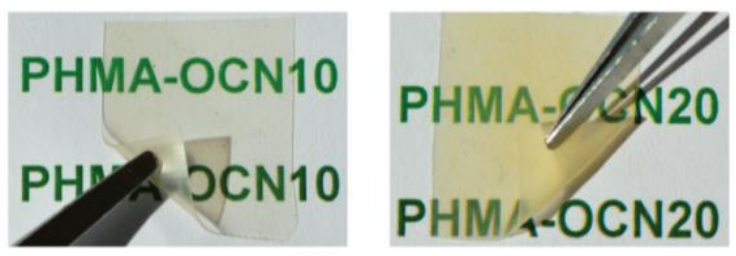

Figure 4. Pictures of (from left to right) OCNs based on PMMA-grafted CNCs and PHMAgrafted CNCs with $10 \mathrm{wt} \%$ and $20 \mathrm{wt} \%$ CNCs.

The transparency of the films was assessed by UV-vis transmittance in the range between 400 and $800 \mathrm{~nm}$ and the results are shown in Figure 5. Neat PMMA and PHMA films transmitted about $97 \%$ of the incident light with very little variation throughout the visible spectrum range. As expected, the transmittance of CNC containing samples was reduced at low 
wavelengths due to scattering effects, but it in the case of the OCNs it approached that of the neat polymers at higher wavelengths. Importantly, across the entire wavelength regime, the OCNs show a considerably higher transparency than the reference composites, supporting the conclusion that aggregation effects are more pronounced in the latter. In contrast to the neat polymers, which were colorless, the CNC-containing films were slightly yellow after compression molding (Figures 4 and S11), on account of thermal degradation of the sulfate half-ester groups present on the surface of the CNCs. ${ }^{47,48}$ We note that when processed at similar temperature, the conventional composites displayed considerably more yellowing than the OCNs, and thus, the processing temperature was reduced for the former (vide supra). This means that the thermal stability of CNCs is increased in the OCNs, arguably due to the presence of polymethacrylate grafts, as previously reported by Börjesson et al. ${ }^{49}$
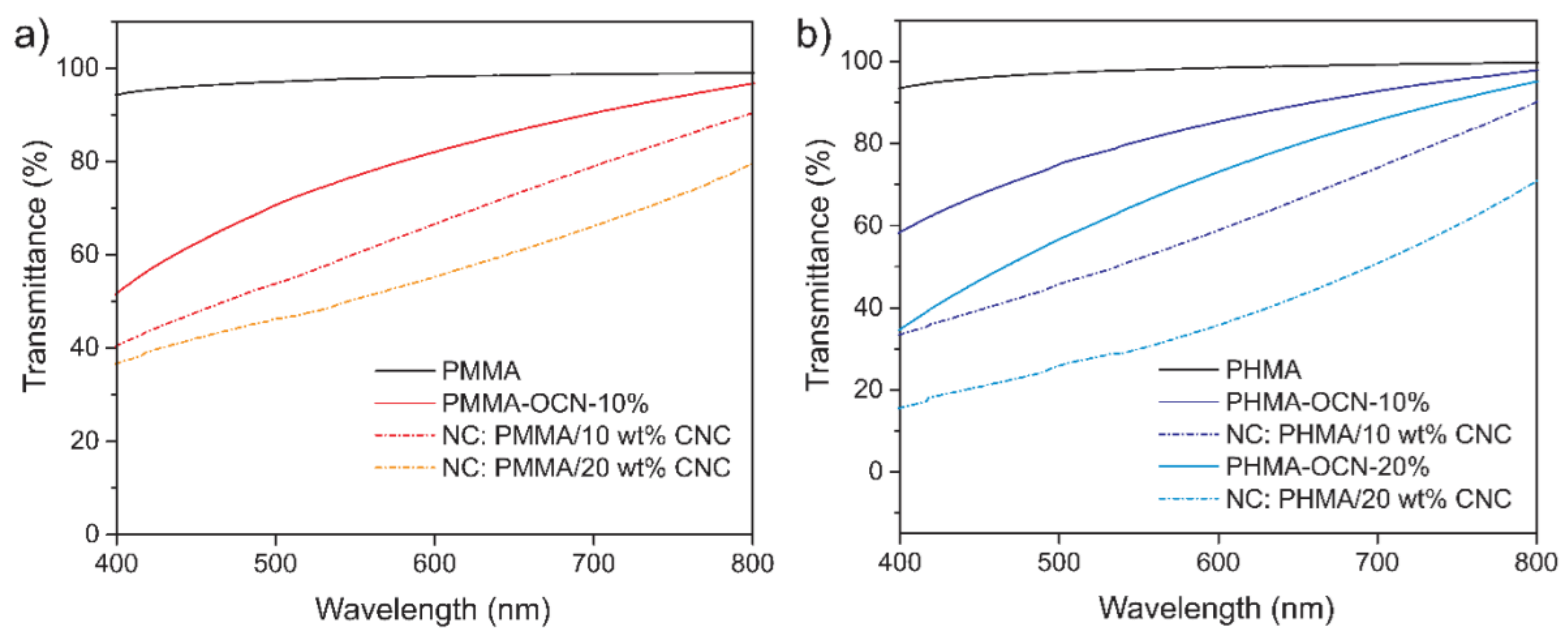

Figure 5. Optical transmittance spectra of neat polymethacrylate (black line), OCNs (solid colored line) and reference composite (dashed line) films. (a) PMMA and (b) PHMA based materials containing 10 or $20 \mathrm{wt} \%$ of CNCs.

In order to evaluate to what extent the crystallinity of the CNCs is impacted upon BPfunctionalization and subsequent graft polymerizations, unmodified and functionalized CNCs were investigated by X-ray diffraction (XRD) experiments (Figures 6 and S12). The 
diffraction peaks observed for a powder sample of unmodified CNCs at $14.86^{\circ}, 16.48^{\circ}$, and $22.72^{\circ}$ are in agreement with literature data, ${ }^{50}$ and can be assigned to the main reflection planes ((110), (110) and (200)) of cellulose I. All diffraction peaks of cellulose I are also present in the powder diffractogram of CNC- $g$-BP. Based on the XRD data, crystallinity indices $\left(\chi_{c}\right)$ of 97 and $90 \%$ were determined for unmodified CNCs and CNC-g-BP by using the method of Segal et al. ${ }^{51}$ As this method only consider the (200) plane, ${ }^{52} \chi_{c}$ does not reflect the actual degree of crystallinity, but the value serves to demonstrate that the crystallinity has been hardly impacted when the CNCs were modified with BP. Unfortunately, $\chi_{c}$ of the HNPs could not be determined due to the presence of pronounced amorphous halos, which result from the polymer grafts and overlap with the crystalline reflections. Nevertheless, the main diffraction peak (200) of cellulose I is visible for all materials albeit with reduced intensity due to the dilution effect of the polymer grafts (Figures 6 and S12).

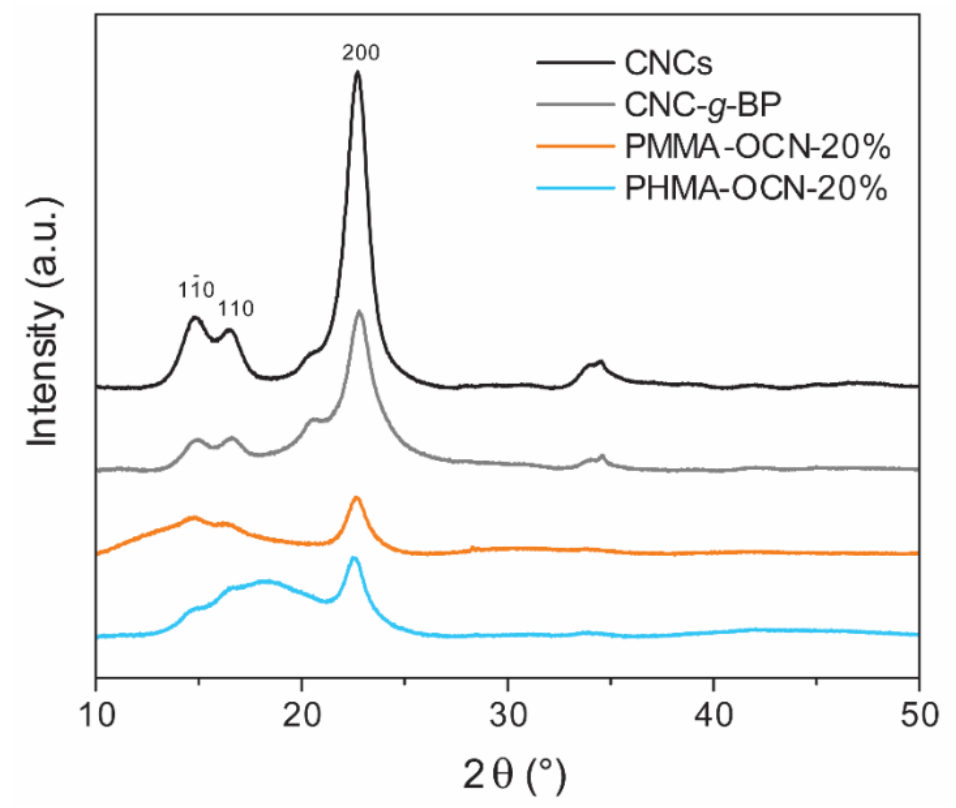

Figure 6. X-ray diffractograms of unmodified $\mathrm{CNCs}, \mathrm{CNC}-g$-BP and $\mathrm{CNC}$-based $\mathrm{OCNs}$ containing $20 \mathrm{wt} \%$ of CNCs (top to bottom). 
Further examination of the OCN films using polarized optical microscopy (POM) revealed highly homogeneous, birefringent materials with larger anisotropic domains for PHMA-OCNs materials (Figure 7a,c). As large-scale phase separation cannot occur and the polymers used are fully amorphous, one can speculate that the anisotropic domains result from the selfassembly of the HNPs in ordered domains. The more pronounced birefringence observed for the PHMA-OCNs materials is consistent with the higher mobility of the low- $T_{\mathrm{g}}$ PHMA, which may allow a better arrangement of the HNPs than in the high- $T_{\mathrm{g}}$ PMMA. POM micrographs of the two-component PMMA/CNC and PHMA/CNC reference nanocomposites containing similar weight fractions of CNCs suggest a lower homogeneity and show less birefringence, presumably, as a result of CNC aggregation (Figure $\mathbf{7 b , d}$ ).
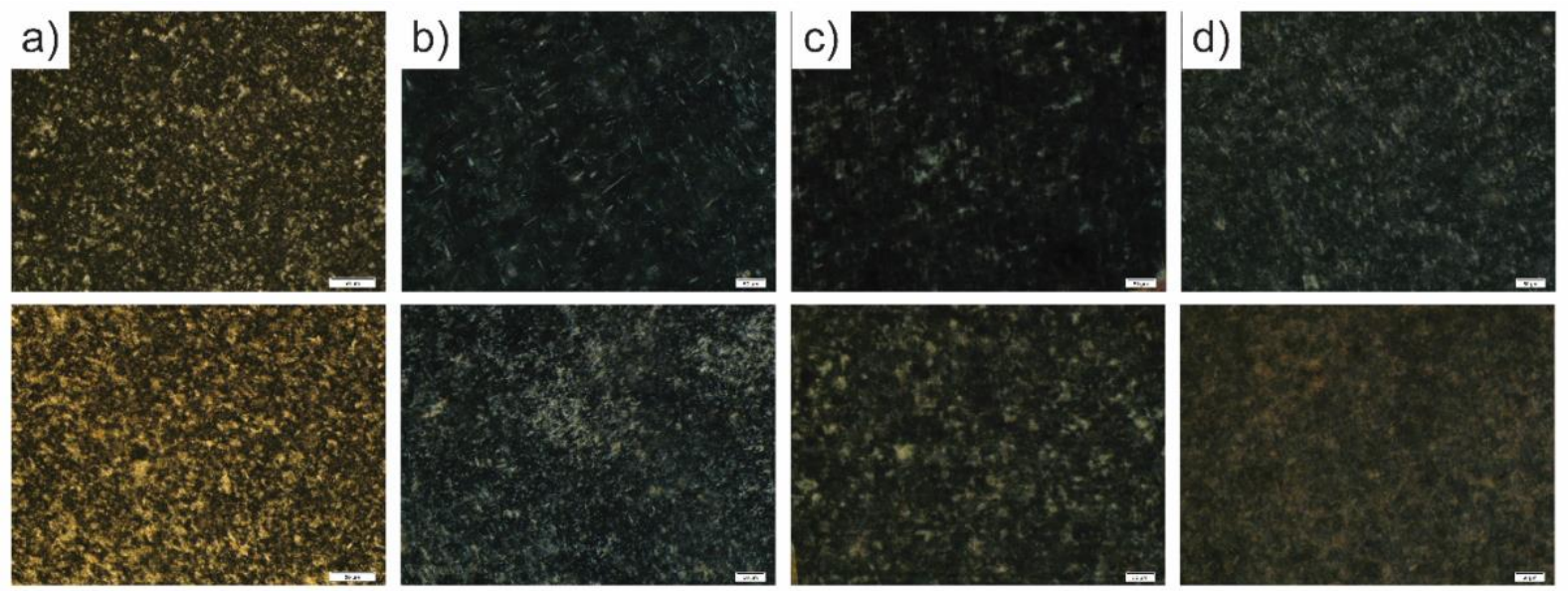

Figure 7. POM micrographs of (a) PMMA-OCNs, (b) PMMA/CNC nanocomposites (c) PHMA-OCNs and (d) PHMA/CNC reference nanocomposites with $10 \mathrm{wt} \%$ (top) and $20 \mathrm{wt} \%$ (bottom) CNCs. Scale bar $=50 \mu \mathrm{m}$.

The thermal properties of the neat polymers, the OCNs, and the two-component reference nanocomposites were investigated by differential scanning calorimetry (DSC) experiments (Figure 8a, Table 2). The DSC traces of the neat PMMA and PHMA (samples used for the DSC experiments were the free polymers produced in the reactions leading to the $10 \% \mathrm{HNPs}$ ) 
exclusively show glass transitions at 98 and $-2{ }^{\circ} \mathrm{C}$. These transitions are also seen in the twocomponent reference nanocomposites. In the case of the OCNs, an increase of the $T_{g}$ of up to $20{ }^{\circ} \mathrm{C}$, relative to the neat polymer, was observed, which may be ascribed to the reduction of the chain mobility due to their grafting on the CNCs surface.

Table 2. Thermal and mechanical properties of the OCNs based on PMMA- and PHMAgrafted CNCs, the neat polymers, and the two-component reference nanocomposites (NC).

\begin{tabular}{|c|c|c|c|c|c|c|c|c|}
\hline \multirow{2}{*}{ Sample } & \multicolumn{2}{|c|}{$\begin{array}{c}T_{g} \\
\left({ }^{\circ} \mathbf{C}\right)\end{array}$} & \multicolumn{2}{|c|}{$\begin{array}{l}\text { Storage modulus } \\
\left(E^{\prime}, \mathrm{MPa}\right)\end{array}$} & \multirow{2}{*}{ 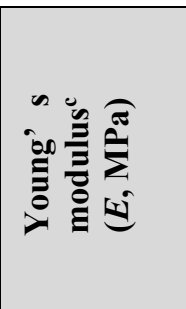 } & \multirow{2}{*}{ 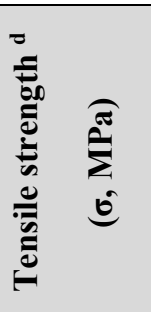 } & \multirow{2}{*}{ 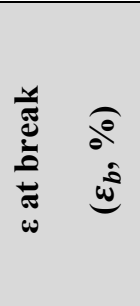 } & \multirow{2}{*}{ 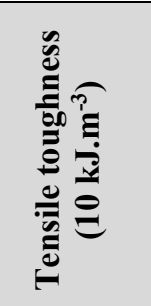 } \\
\hline & Uூ & $\sum_{i}^{e}$ & $-60^{\circ} \mathrm{C}$ & $25^{\circ} \mathrm{C}$ & & & & \\
\hline Neat PMMA & 98 & 95 & $4738 \pm 58$ & $3509 \pm 62$ & $2029 \pm 64$ & $67 \pm 4$ & $5.3 \pm 0.4$ & $297 \pm 35$ \\
\hline PMMA-OCN-10\% & 119 & 124 & $4728 \pm 815$ & $3168 \pm 251$ & $2026 \pm 109$ & $39 \pm 4^{\mathrm{e}}$ & $25 \pm 1$ & $759 \pm 53$ \\
\hline $\begin{array}{l}\text { NC: } \quad \text { PMMA } / 10 \% \\
\text { CNC }\end{array}$ & 96 & 95 & $5482 \pm 213$ & $3658 \pm 107$ & $1903 \pm 113$ & $36 \pm 4$ & $2.8 \pm 0.3$ & $61 \pm 13$ \\
\hline PMMA-OCN-20\% & 115 & 136 & $5227 \pm 505$ & $3658 \pm 293$ & $2560 \pm 150$ & $38 \pm 2$ & $1.7 \pm 0.2$ & $34 \pm 7$ \\
\hline $\begin{array}{l}\text { NC: } \quad \text { PMMA } / 20 \% \\
\text { CNC }\end{array}$ & 95 & 93 & $6092 \pm 115$ & $4319 \pm 50$ & $2371 \pm 173$ & $37 \pm 5$ & $2.2 \pm 0.6$ & $57 \pm 16$ \\
\hline Neat PHMA & -2 & 1 & $1770 \pm 373$ & $15 \pm 4$ & $7.4 \pm 0.4$ & $1.8 \pm 0.2$ & $425 \pm 23$ & $420 \pm 71$ \\
\hline PHMA-OCN-10\% & -1 & 4 & $2160 \pm 35$ & $59 \pm 2$ & $28 \pm 1$ & $6.8 \pm 0.6$ & $201 \pm 22$ & $801 \pm 131$ \\
\hline $\begin{array}{l}\text { NC: PHMA } / 10 \\
\text { wt } \% \text { CNC }\end{array}$ & -2 & 3 & $2220 \pm 79$ & $48 \pm 18$ & $15 \pm 1$ & $2.6 \pm 0.2$ & $545 \pm 29$ & $\begin{array}{l}1077 \pm \\
109\end{array}$ \\
\hline PHMA-OCN-20\% & -3 & 11 & $2709 \pm 208$ & $317 \pm 30$ & $99 \pm 3$ & $10.4 \pm 0.5$ & $79 \pm 7$ & $528 \pm 66$ \\
\hline $\begin{array}{l}\text { NC: PHMA } / 20 \\
\text { wt } \% \text { CNC }\end{array}$ & -2 & 3 & $2748 \pm 137$ & $45 \pm 3$ & $30 \pm 2$ & $2.9 \pm 0.2$ & $248 \pm 43$ & $560 \pm 121$ \\
\hline
\end{tabular}

${ }^{a}$ Determined by DSC. ${ }^{b}$ Determined from the maxima of the shear loss modulus peaks (Figure S13). ${ }^{c}$ Determined from the slope of the initial elastic region (between $0.1 \%$ and $0.25 \%$ ) of the

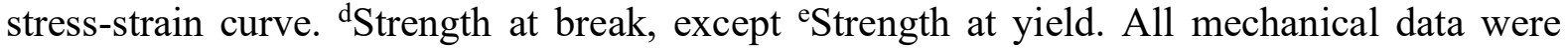
established by uniaxial tensile tests.

The thermomechanical properties of all materials were investigated by dynamic mechanical analysis (DMA). The results are presented in Figure $\mathbf{8 b - c}$ and $\mathbf{S 1 3}$, and the most relevant 
mechanical data established by these experiments are compiled in Table 2. All samples display a clear transition from a glassy to a viscous or rubbery regime around the $T_{g}$ observed by DSC analysis. In the case of the PMMA-based materials (Figure 8b), the shear storage modulus $\left(E^{\prime}\right)$ in the glassy regime increases slightly upon introducing CNCs from 4.7 GPa (neat PMMA at $-60{ }^{\circ} \mathrm{C}$ ) to 5.2 (PMMA-OCN-20\%) to $6.1 \mathrm{GPa}$ (PMMA/20\% CNC nanocomposite). As suggested by the DSC results, the $T_{g}$ 's of the PMMA-OCNs, based on the maxima of the shear loss modulus peaks shown in Supporting Figure S13 and Table 2, are ca. $30^{\circ} \mathrm{C}$ higher than those of the conventional composites. As a result, the PMMA-OCNs display at $200{ }^{\circ} \mathrm{C}$ an $E$ ' of 1.4 and $7.9 \mathrm{MPa}$, whereas the reference composites are barely measurable. The behavior of the PHMA-based materials is qualitatively similar, with a lower $E^{\prime}$ and a higher relative reinforcing effect at all temperatures and a much wider (observable) rubbery regime for the OCNs (Figure 8c, Table 2). The PHMA-OCNs display an extended rubbery plateau, arguably due to massive chain entanglements between the brush-like particles, while the reference composites transition into a viscous melt. In this case, no difference of the low-temperature $E$, was observed between the PHMA-OCNs and the corresponding two-component reference nanocomposite. 
a)
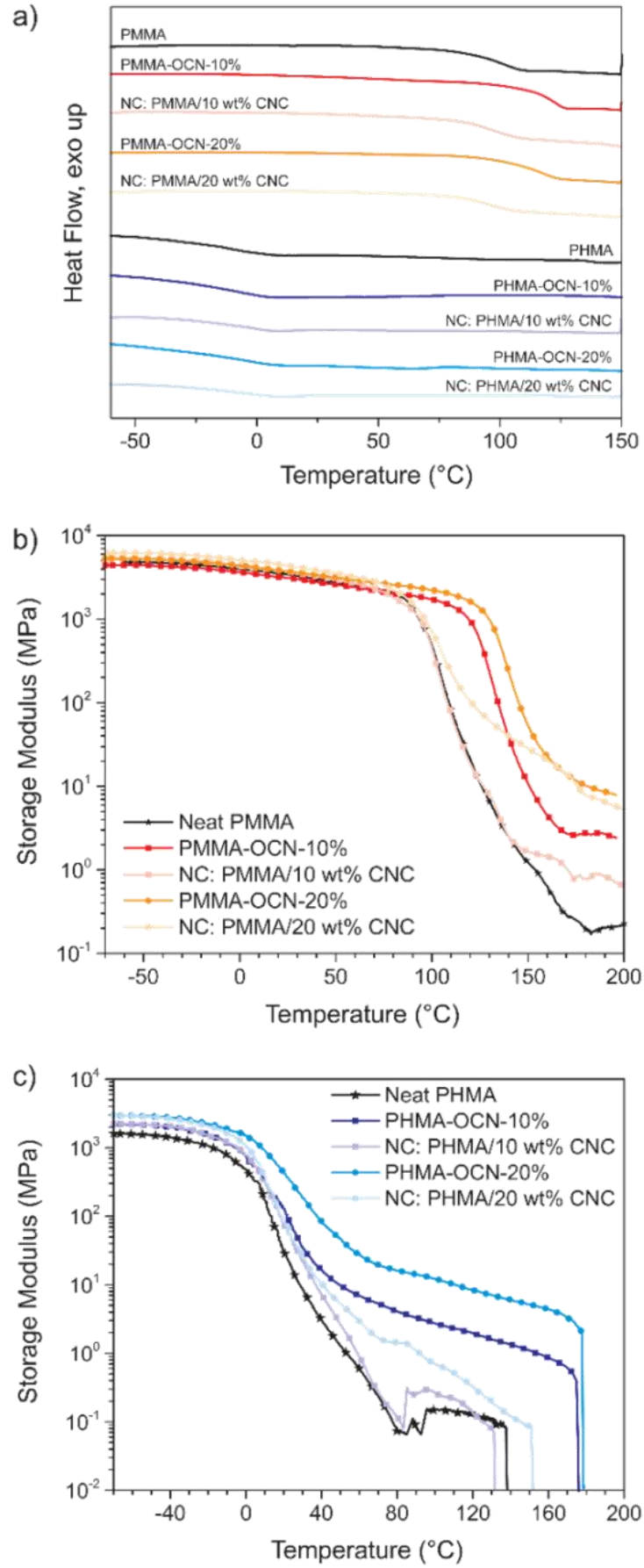

Figure 8. (a) DSC (a) and DMA (b, c) traces of the OCNs based on PMMA- and PHMAgrafted $\mathrm{CNCs}$, the neat polymers, and the two-component reference nanocomposites (NC).

In order to exclude that the improved mechanical properties of the OCNs, in particular the extensions of the rubbery plateaus, are related to covalent-cross-linking between the HNPs during melt-processing, we tested if the OCN films could be redispersed. Gratifyingly, all OCN 
films fully dispersed when $0.3 \mathrm{wt} \%$ mixtures were stirred in dichloromethane (DCM), chloroform, THF, or acetone for 5 to $20 \mathrm{~min}$ (Figure 9). We note that neither of these solvents is useful to disperse dried unmodified CNCs. When the concentrations of OCNs were increased to higher than $1 \mathrm{wt} \%$, longer times were needed to reach complete dispersion. The absence of chemical cross-linking is further supported by additional DMA experiments with the PMMAOCNs, in which the temperature range was extended to $250{ }^{\circ} \mathrm{C}$ (Figure S14). For both compositions the storage modulus continues to drop is the temperature was raised above 200 ${ }^{\circ} \mathrm{C}$, while no changes in the transition slope or relaxation in the transition region, which are characteristic of cross-linked networks, ${ }^{53}$ could be observed.

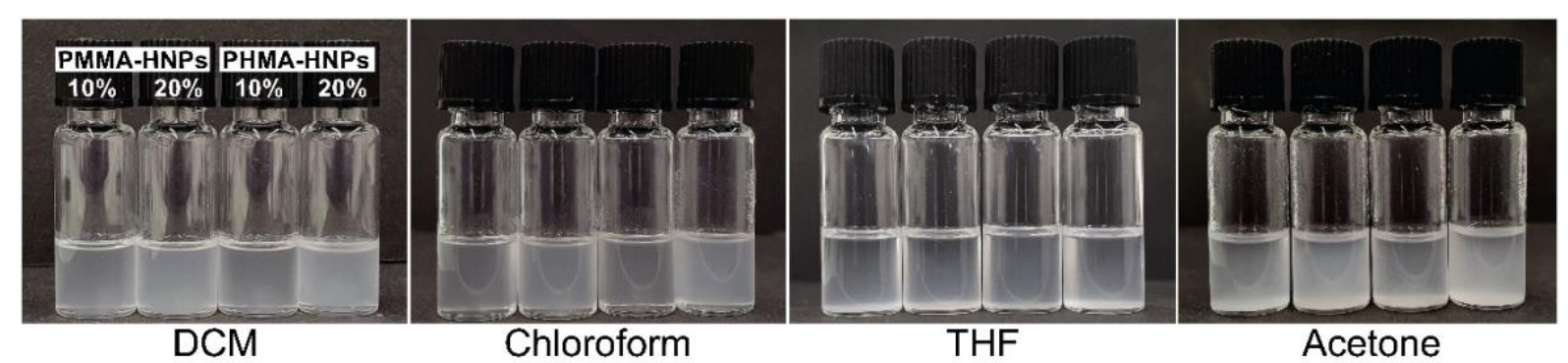

Figure 9. Suspensions of PMMA- and PHMA-HNPs containing 10 and $20 \mathrm{wt} \%$ of CNCs (from left to right) dispersed in different solvents.

The tensile properties of films of the OCNs, the parent polymers, and the reference nanocomposites were investigated by uniaxial tensile tests at room temperature. Figure 10 shows the stress-strain curves and relevant mechanical data extracted from these experiments are summarized in Table 2. The tensile properties of PMMA are known to depend on the strain rate $^{54}$ and temperature, ${ }^{55}$ and the yield strength increases with the strain rate. ${ }^{54}$ The stress-strain curves of PMMA, PMMA-OCNs films, and PMMA/CNC conventional nanocomposites, recorded at a low strain rate of $1.3 \mathrm{~mm} / \mathrm{min}$ at $25^{\circ} \mathrm{C}$, are presented in Figure 10a. PMMA and 
the conventional composites all show an initial elastic regime, characterized by a Young's modulus of the order of $2 \mathrm{GPa}$, followed by brittle fracture at low strain. The tensile strength (67 $\mathrm{MPa})$ and elongation at break $(5.3 \%)$ of PMMA isolated from the supernatant of the PMMA-HPN-10\% synthesis are in good agreement with the data reported elsewhere. ${ }^{55,56}$ The two-component PMMA/CNC reference nanocomposites with 10 or 20\% CNCs (Young's modulus of 1.9 and $2.4 \mathrm{GPa})$ are embrittled and fracture at lower strain (2.8 and $2.2 \%)$ and stress (36 and $37 \mathrm{MPa}$ ) than the neat PMMA. Interestingly, the PMMA-OCN-10\% displays an equivalent Young's modulus (i.e., $2 \mathrm{GPa}$ ) but a very different deformation behavior, yielding at low strain (3.3\%) and exhibiting plastic deformation up to a fracture strain of $25 \%$. While the maximum strength (observed at the yield point) of $39 \mathrm{MPa}$ is reduced in comparison to the neat PMMA, the toughness of the PMMA-OCN-10\% is doubled. The PMMA-OCN-20\% films were stiffer and more brittle than PMMA-OCN-10\%, and show a deformation behavior that is similar to that of the PMMA/CNC reference nanocomposite.

In contrast to the glassy PMMA, PHMA displays a $T_{g}$ below room temperature so that the mechanical properties at ambient temperature are dominated by chain entanglements and are consequently highly strain-rate dependent; tensile tests were thus performed with a high strain rate of $130 \mathrm{~mm} / \mathrm{min}$. As expected, the stress-strain curves of the neat PHMA exhibit a very high extensibility (425\%) with low Young's modulus (7.4 MPa) and tensile strength (1.8 MPa) (Figure 10b). In the case of the reference nanocomposites formed by combination of PHMA and unmodified CNCs, the tensile strength increased to 2.6 and 2.9 MPa, and the Young's modulus to 15 and $30 \mathrm{MPa}$, upon incorporation of 10 or $20 \mathrm{wt} \% \mathrm{CNCs}$, while the elongation at break remained very high (545 and 248\%). The PHMA-OCN-10\% and 20\% samples are much stiffer (Young's moduli $=28$ and $99 \mathrm{MPa}$ ) and stronger (tensile strength $=6.8$ and 10.4 $\mathrm{MPa}$ ) than the neat PHMA and also the conventional PHMA/CNC nanocomposites, and the extensibility remains rather high (201 and 79\%, respectively). Thus, the data show clearly that 
the mechanical properties of the new OCNs in the rubbery state are governed by the unique architecture that involves a separation of the CNCs through the attached polymer surface layer, while stress transfer among the particles is enabled by chain entanglements between them, which also depend on the grafting density and the degree of polymerization. ${ }^{57}$
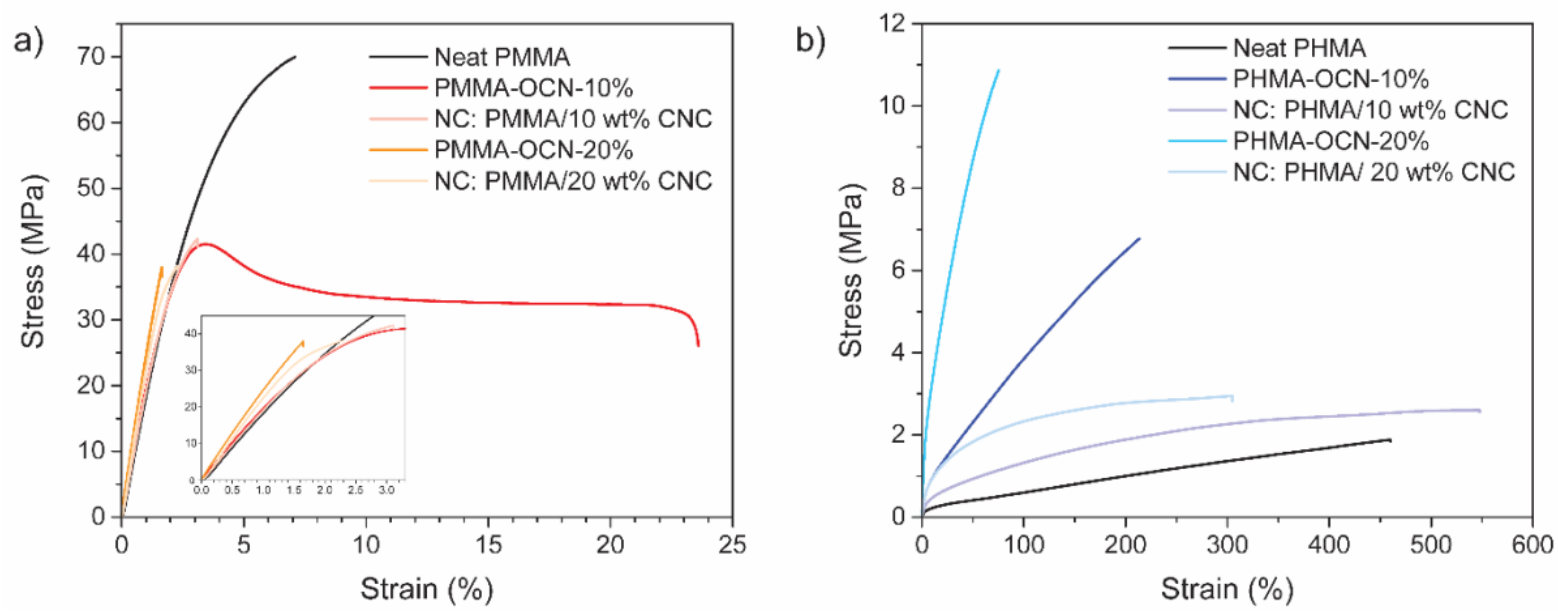

Figure 10. Stress-strain curves of OCNs based on (a) PMMA- and (b) PHMA-grafted CNCs, the neat polymers, and the two-component reference nanocomposites (NC). Data were collected at room temperature.

In order to investigate the elastic behavior of the PHMA-OCNs in more detail, tensile creep tests were performed on PHMA-OCNs and the corresponding two-component nanocomposites under two constant forces. Samples were initially loaded with $0.4 \mathrm{MPa}$ or $1.3 \mathrm{MPa}$ at room temperature using the same strain rate as for static tensile tests (i.e., $130 \mathrm{~mm} / \mathrm{min}$ ). The applied stress was then kept constant for 12 min and the change in length was recorded during this time. The data thus collected is reported as creep strain versus time (Figure 11). Interestingly, while most samples show considerable relaxation, PHMA-OCN-20\% shows no significant creep at either of the applied loads. An analysis of the traces reveals creep moduli $\left(E_{c}\right)$ of 16 $\mathrm{kPa}$ and $24 \mathrm{kPa}$ for PHMA-OCN-20\% at fixed loads of 0.4 and $1.3 \mathrm{MPa}$, respectively. Also PHMA-OCN-10\% show very little deformation under a load of $0.4 \mathrm{MPa}\left(E_{c}=4.3 \mathrm{kPa}\right)$ but 
creep becomes more pronounced when the constant loading is increased to $1.3 \mathrm{MPa}\left(E_{c}=2.0\right.$ $\mathrm{kPa}$ ). An inspection of the graphs show strikingly that both two-component reference nanocomposites show much more remarkable creep than the PHMA-OCNs. The twocomponent nanocomposite $\mathrm{PHMA} / 10 \% \mathrm{CNC}$ is the one that creeped the most from all the materials under study, with $E_{c}$ values of 0.9 and $2.6 \mathrm{kPa}$ for loads of 0.4 and $1.3 \mathrm{MPa}$, respectively, while the higher $\mathrm{CNC}$ content in PHMA/20\%CNC reduced creep a bit. The significantly reduced creep seen for the OCNs is likely related to the fact that massive "parallel" chain entanglements between the brush-like particles are at play, and confirms once more that the reinforcement achieved by fabrication OCNs is improved in comparison with conventional two-component nanocomposite due to their architectural design.
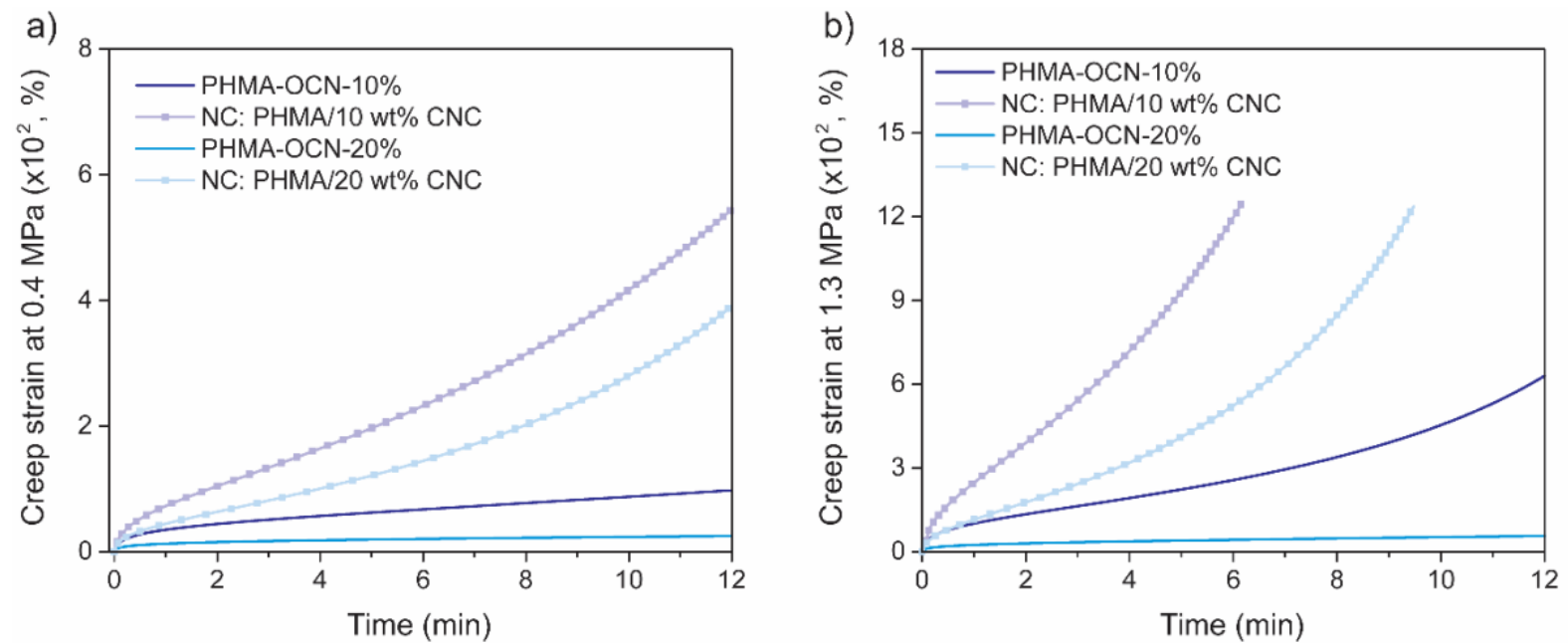

Figure 11. Tensile creep curve of PHMA-OCNs materials and the corresponding twocomponent reference nanocomposites at a constant loading of (a) $0.4 \mathrm{MPa}$ and (b) $1.3 \mathrm{MPa}$ at room temperature. 


\section{CONCLUSIONS}

In summary, we have shown that polymethacrylate-grafted CNCs, in which the nature and weight fraction of the polymer graft can be easily controlled, are readily accessible functionalizing CNCs with benzophenone moieties and surface-photoinitiated polymerization with PMMA or PHMA. OCNs formed by the assembly of the HNPs -made display properties that are clearly different from those of the corresponding two-component reference nanocomposites. The PMMA-OCNs show the typical behavior of rigid, glassy polymers, with PMMA-OCN-10\% being much tougher and PMMA-OCN-20\% much stiffer than the neat PMMA and the two-component reference nanocomposites. By contrast, the PHMA-OCNs are rubbery at room temperature, and display a rather high extensibility, while offering higher stiffness and strength than the neat PHMA and the conventional PHMA/CNC nanocomposites. In both platforms, the rubbery regime is considerably extended, and the data suggest that stress transfer, at least above $T_{\mathrm{g}}$, is dominated by chain entanglements between the hairy CNCs. This approach leads to a significant improvement of the mechanical properties of OCNs containing low $T_{\mathrm{g}}$ grafts. The OCNs are also highly transparent, which supports the conclusion that CNC aggregation can be avoided. The design approach should be general and applicable to other hybrid materials in which a significant mechanical performance is required without compromising the homogeneity of the final product.

\section{EXPERIMENTAL SECTION}

Materials. 4-Hydroxybenzophenone, hexamethylene diisocyanate (HMDI), dibutyltin dilaurate (DBTDL), chlorotrimethylsilane, sulfuric acid $\left(\mathrm{H}_{2} \mathrm{SO}_{4}, 95-98 \%\right)$ and pentane were purchased from Sigma-Aldrich and were used as received. Anhydrous DMF and THF (from Acros), and deuterated chloroform (from Cambridge Isotope Laboratories, Inc.) were used as 
received. Methyl methacrylate (MMA) and hexyl methacrylate (HMA) were passed through an activated basic (Brockmann I) alumina column prior to use, in order to remove the inhibitor. Cotton-based CNCs, with an average length of $117 \pm 28 \mathrm{~nm}$ and average width of $15 \pm 2 \mathrm{~nm}$ (aspect ratio of $8 \pm 2$, both determined by image analysis of TEM images), were extracted from Whatman no. 1 filter paper by sulfuric acid hydrolysis following a previously published protocol. ${ }^{20}$ The aqueous $\mathrm{CNC}$ dispersion that resulted from the process was dried via lyophilization using a VirTis BenchTop 2K XL lyophilizer with a condenser temperature of $78^{\circ} \mathrm{C}$.

Methods. A Bruker Avance DPX 400 spectrometer was utilized to record ${ }^{1} \mathrm{H}$ NMR (400 $\mathrm{MHz})$ and ${ }^{13} \mathrm{C}$ NMR (100 MHz) spectra at 297.2 K. Data were analyzed with the MestReNova software (v 11.0), and all chemical shifts $(\delta)$ are reported in parts per million (ppm) relative to the signal of tetramethylsilane (TMS), although referencing was carried out on the basis of the residual solvent signal, i.e., deuterochloroform $\left(\mathrm{CDCl}_{3}, \delta_{\mathrm{H}} 7.26, \delta_{\mathrm{C}} 77.16\right)$.

Fourier-transform infrared (FTIR) spectra were recorded on a PerkinElmer Spectrum 65 spectrometer equipped with an attenuated total reflection (ATR) setup. All spectra were collected in the wavelength range between 600 and $4000 \mathrm{~cm}^{-1}$ after 30 continuous scans.

$\mathrm{UV}$-vis absorption spectra in the wavelength range between 250 and $350 \mathrm{~nm}$ were recorded on a Shimadzu UV-2401 PC spectrophotometer in anhydrous DMF using quartz cuvettes with a path length of $1 \mathrm{~cm}$. The transparency of films of a thickness of about $0.2 \mathrm{~mm}$ was measured using the same spectrophotometer above mentioned in air using a wavelength range between 400 and $800 \mathrm{~nm}$. Films were cut to about 9x20 mm and were placed onto a quartz slide prior to measurement. 
The determination of $\mathrm{C}, \mathrm{H}, \mathrm{N}$ and $\mathrm{S}$ contents was done by elemental analysis, which was performed at the ETH Zurich, Switzerland. The combustion products resulting from the sample digestion, i.e., $\mathrm{CO}_{2}, \mathrm{H}_{2} \mathrm{O}$, were quantified by infrared spectroscopy to determine $\mathrm{C}$ and $\mathrm{H}$ contents, respectively. $\mathrm{N}$ and $\mathrm{S}$ were measured as $\mathrm{N}_{2}$ and $\mathrm{SO}_{2}$ by quantification of their thermal conductivity upon burning the sample at $1000^{\circ} \mathrm{C}$.

Mass spectrometry (MS) data for small molecules were provided by external analysis services at the Chemistry Department of the University of Fribourg. Electrospray ionization mass spectrometry (ESI-MS) data were collected on a Bruker FTMS 4.7 T BioAPEXII equipped with a ComiSource 1.0and run in the positive mode.

An Agilent 1200 series HPLC system was used for size exclusion chromatography (SEC) experiments. The instrument was equipped with an Agilent PLgel mixed guard column (particle size $=5 \mu \mathrm{m}$ ) and two Agilent PLgel mixed-D columns (ID $=7.5 \mathrm{~mm}, \mathrm{~L}=300 \mathrm{~mm}$, particle size $=5 \mu \mathrm{m}$ ) and experiments were conducted at a flow rate of $1.0 \mathrm{~mL} / \mathrm{min}$, at $30{ }^{\circ} \mathrm{C}$, and with THF as the eluent. Signals were recorded using an Optilab REX interferometric refractometer, a UV detector (Agilent 1200 series), and a miniDawn TREOS light scattering detector (Wyatt Technology Corp.). A calibration with narrow molecular weight poly(methyl methacrylate) standards (from 550 to $265300 \mathrm{~g} / \mathrm{mol}$ ) was utilized and Astra software (Wyatt Technology Corp.) was used to analyze the data.

Films of the OCNs having a thickness of $0.2 \mathrm{~mm}$ were prepared by compression molding the HNPs in a Carver CE press between two metallic plates of $15 \times 15 \mathrm{~cm}$ and using $0.2 \mathrm{~mm}$ spacers and 7 metric tons of pressure for different times ( $5 \mathrm{~min}$ or $10 \mathrm{sec})$ and temperatures $\left(160{ }^{\circ} \mathrm{C}\right.$ or $130{ }^{\circ} \mathrm{C}$ ) according to the material under press (see Synthesis Section for more details). Polymethacrylate/CNC nanocomposites with $10 \mathrm{wt} \%$ or $20 \mathrm{wt} \%$ of CNCs were prepared by casting from THF. Thus, the dry CNCs were dispersed in THF $(10 \mathrm{mg} / \mathrm{mL})$ by stirring for 20 
$\mathrm{h}$ at room temperature before the mixture was sonicated for $3 \mathrm{~h}$ in a Sonoswiss $\mathrm{SW} 3 \mathrm{H}$ ultrasonic unit. The CNC dispersion was then combined with a solution of the polymethacrylate (PMMA or PHMA) isolated from the supernatant of the corresponding HNPs in THF (30 and $40 \mathrm{mg} / \mathrm{mL}$, respectively), which had been stirred at room temperature for $24 \mathrm{~h}$ and heated the last hour at $50{ }^{\circ} \mathrm{C}$. The volume of the $\mathrm{CNC}$ dispersion was controlled in order to achieve the desired CNCs concentration in the nanocomposites. The final mixture was stirred for $10 \mathrm{~min}$ at $50{ }^{\circ} \mathrm{C}$, sonicated again for $30 \mathrm{~min}$ at room temperature in a Sonoswiss SW3H ultrasonic unit, before it was finally cast into a Teflon Petri dish with an inner diameter of $10 \mathrm{~cm}$. The solvent was evaporated in an oven at $50{ }^{\circ} \mathrm{C}$ for $48 \mathrm{~h}$. Finally, the dried films were compression molded in a Carver CE press between Kapton sheets at $130{ }^{\circ} \mathrm{C}$ or Teflon sheets at $100{ }^{\circ} \mathrm{C}$ (for PMMA and PHMA, respectively) for 30 or $15 \mathrm{sec}$. A pressure of 7 metric tons was applied and spacers were used to adjust the film thickness to $0.2 \mathrm{~mm}$. To minimize moisture absorption the films were then stored at ambient temperature over drierite in a desiccator, until they were used.

X-ray diffraction (XRD) measurements were carried out with a XRD Rigaku Ultima IV. Powder and film samples were loaded onto a rectangular glass sample holder and scans were obtained using $\mathrm{CuK} \alpha$ radiation $(\lambda=1.5401 \AA)$ under a voltage of $30 \mathrm{kV}$ with a scan rate of 0.5 $\circ / \mathrm{min}$. The $2\left(\right.$ parameter was varied between $10^{\circ}$ and $50^{\circ}$ in steps of $0.02^{\circ}$.

Differential scanning calorimetry (DSC) measurements were performed under $\mathrm{N}_{2}$ using a Mettler-Toledo STAR system operating at heating/cooling rates of $10^{\circ} \mathrm{C} / \mathrm{min}$ in the range from $-80{ }^{\circ} \mathrm{C}$ to $150^{\circ} \mathrm{C}$ and two heating/cooling cycles were routinely applied. The $T_{g}$ values were determined from the second heating scan, and the midpoint of the transition was reported.

The mechanical properties of the neat polymethacrylates, the OCNs, and the two-component reference nanocomposites were studied using a TA Instrument, DMA Q800. The experiments were performed in a tensile mode with a strain amplitude of $15 \mu \mathrm{m}$, at a frequency of $1 \mathrm{~Hz}$ and 
a heating rate of $5{ }^{\circ} \mathrm{C} / \mathrm{min}$ in the temperature range from $-70{ }^{\circ} \mathrm{C}$ to $200{ }^{\circ} \mathrm{C}$ or $250{ }^{\circ} \mathrm{C}$ using a film tension clamp. The testing specimens were cut in rectangular shape of approximate dimensions: $15 \mathrm{~mm} \times 3.5 \mathrm{~mm} \times 0.2 \mathrm{~mm}$. The data reported were the average of 3 independent measurements and all errors were standard deviations. The storage moduli were determined with a $\pm 1 \%$ precision according to the manufacturer specifications. Standard deviations are reported as the variance in a set of samples compared to the average (mean) of the measurement.

Microtensile specimens with an initial overall length of $38 \mathrm{~mm}$ in dog-bone shape and of approx. $0.2 \mathrm{~mm}$ thickness were cut using a die-cutting tool according to ASTM D1708 from compression-molded films of the neat polymethacrylates, the OCNs, and the two-component reference nanocomposites. All materials were dried under vacuum for at least 2 days before testing. The testing procedure followed the standard method described in ASTM D1708, but no extensometer was used. Tensile measurements were performed at room temperature using a static material testing machine from Zwick/Roell with a $200 \mathrm{~N}$ Xforce HP load cell. The reported data are averages of 5 independent measurements and all errors quoted are standard deviations. The strain rate was 1.3 and $130 \mathrm{~mm} / \mathrm{min}$ for PMMA and PHMA containing materials, respectively. The Young's modulus of materials was determined from the slope of the linear portion of the stress-strain curve, more specifically between 0.1 and $0.25 \%$ of strain. This value is subject to accuracy limitations due to the type of specimen used during the measurement (i.e., microtensile specimens). In addition, tensile creep measurements were performed at room temperature using the same machine and specimen type than for uniaxial tensile tests. The measurements were executed at levels of stress (i.e., 0.4 and 1.3 MPa) with an initial strain rate of $130 \mathrm{~mm} / \mathrm{min}$. Once the loading was achieved, it was maintained constant and the variations in the length of the specimen were recorded for $12 \mathrm{~min}$. In order to avoid a significant variation in the applied load, the variation of about $\pm 2 \%$ was pre-stablished. The 
reported data are average of 3 independent measurements and all errors quoted are standard deviations. The creep modulus was determined as the ratio between the initial stress and the final strain. The confidence interval of all measurements was $0.13 \%$ according to the last calibration of the instrument (September, 2019), performed under DIN EN ISO 75001 standard. Standard deviations are reported as the variance there is in a set of samples compared to the average (mean) of the measurement.

The average length and width of unmodified CNCs were determined by images analysis of transmission electron microscopy (TEM) micrographs, using a $0.001 \mathrm{wt} \%$ dispersion of CNCs, due to the well-known tip broadening effects on AFM images. The analysis resulted a length of $117 \pm 28$ and a width of $15 \pm 2$ (Figure S1). It is important to note that even in a welldispersed suspension of neat CNCs, they are commonly found by pairs, which may alter the width value here determined. Nevertheless, the particle size analysis of CNCs by AFM and TEM are well-stablished methods to quantitatively determining the dimensions of CNCs. The average height of unmodified CNCs were obtained from images recorded by atomic force microscopy (AFM). First, a freshly pealed mica was functionalized with an aqueous solution of poly-L-lysine by drop-casting $(40 \mu \mathrm{L})$. After $5 \mathrm{~min}$, the poly-L-lysine was washed off with milli-Q water and dried under a flow of nitrogen. Aqueous CNCs suspensions $(0.001 \mathrm{wt} \%, 40$ $\mu \mathrm{L}$ ) were then spin-coated onto the functionalized mica surface at $2000 \mathrm{rpm}$ and subsequently dried under nitrogen flow. The images were acquired with a JPK NanoWizard II, in tapping mode with PPP-NCSTR probes at room temperature and using silicon cantilever. Images were recorded with $2048 \times 2048$ pixels of resolution in a $5 \times 5 \mu \mathrm{m}$ square. The analysis resulted a height of $6 \pm 1 \mathrm{~nm}$ (Figure S15).

TEM micrographs of unmodified CNCs, CNC-g-BP, and the HNPs were recorded on a Tecnai Spirit transmission electron microscope (TEM, FEI/ThermoFischer, Hillsboro, Oregon, 
United States) operating at $120 \mathrm{kV}$ using a 2k Veleta camera (Olympus, Shinjuku, Tokyo, Japan). A bandpass filter (ImageJ, settings 80/3) was applied to reduce noise. $5 \mu \mathrm{L}$ of sample suspensions (i.e., $0.01 \mathrm{wt} \%$ of CNCs in water or CNC-g-BP in DMF and $0.3 \mathrm{wt} \%$ of HNPs in chloroform) were spin-coated at $2000 \mathrm{rpm}$ on a previously plasma treated carbon film 300 mesh copper TEM-grids (Electron microscopy science, USA). The samples were allowed to dry at $60{ }^{\circ} \mathrm{C}$ overnight before imaging. Optical microscopy images were captured with an Olympus BX51 microscope equipped with a DP72 digital camera and between crossed linear polarizers. Photographs were taken with a Nikon D7100 digital camera fitted with an AF-S DX ZoomNIKKOR 18-135 mm lens.

The specific surface area (SSA) of CNCs was determined as follows. Considering the average dimensions of cotton CNCs determined by TEM and AFM images, i.e., a width of 15 $\mathrm{nm}$ and height of $6 \mathrm{~nm}$, and assuming their density to be equivalent to $1.5 \times 10^{6} \mathrm{~g} / \mathrm{m}^{3}$ as reported elsewhere, ${ }^{58}$ and an ellipsoidal cross-section model of cotton CNCs, the SSA was calculated to be $174 \mathrm{~m}^{2} / \mathrm{g}$. This value is in good agreement with the SSA reported by Lin and Dufresne of $191.2 \mathrm{~m}^{2} / \mathrm{g}$ using same cross-section model..$^{58}$

The surface charge density of cotton CNCs was determined by conductometric titration according to a well-known procedure. ${ }^{33}$ First, a dialyzed suspension of $0.71 \mathrm{wt} \%$ CNCs was passed through a previously washed strong acid cation exchange resin (DOWEX Marathon C hydrogen form, Sigma-Aldrich) column. Then, $20 \mathrm{~mL}$ of the resultant suspension, containing $1 \mathrm{mM} \mathrm{NaCl}$, was titrated against $0.01 \mathrm{M}$ standardized $\mathrm{NaOH}$ solution using a SevenCompact Duo S213 pH/conductivity meter. The first equivalence point was assigned to the sulfate half ester groups content (115 mmol $\left.\mathrm{R}-\mathrm{OSO}_{3} \mathrm{H} / \mathrm{kg} \mathrm{CNCs}\right)$, while the second equivalence point corresponds to the carboxylic groups (21 mmol/kg CNCs) (Figure S2). 


\section{Synthesis.}

Synthesis of 4-benzoylphenyl (6-isocyanatohexyl) carbamate (1). Compound 1 was synthesized according to the procedure reported by Biyani et al. with some modifications. ${ }^{34}$ In a $100 \mathrm{~mL}$ three-necked round-bottom amber flask equipped with a reflux condenser and a stirring bar, 4-hydroxybenzophenone (10.0 g, $50.5 \mathrm{mmol})$ and HMDI (40.5 mL, $252 \mathrm{mmol})$ were added. The reaction mixture was stirred at $100{ }^{\circ} \mathrm{C}$ under $\mathrm{N}_{2}$ atmosphere for $24 \mathrm{~h}$. After cooling the reaction mixture to room temperature, it was added drop-wise to an excess of cold pentane $(150 \mathrm{~mL})$ under mechanical stirring in order to remove any excess of residual HMDI. After 5 min of stirring, the resulting suspension was allowed to settle down and the supernatant (pentane) was decanted. The remaining product was dissolved in $20 \mathrm{~mL}$ of ethyl acetate, the solution was filtered, and the liquid phase concentrated under reduced vacuum. The above process was repeated three times in order to remove all residual HMDI. The resulting white viscous liquid was dried in a thermal bath at $50{ }^{\circ} \mathrm{C}$ and under high vacuum for 2 days to obtain 1 as a white oily product $(12.9 \mathrm{~g}, 70 \%) .{ }^{1} \mathrm{H} \mathrm{NMR}\left(\mathrm{CDCl}_{3}, 400 \mathrm{~Hz}\right):{ }^{\mathrm{TM}}=7.78-7.84(\mathrm{~m}, 4 \mathrm{H}$, $\operatorname{Ar} H), 7.56-7.61(\mathrm{~m}, 1 \mathrm{H}, \operatorname{Ar} H), 7.46-7.50(\mathrm{~m}, 2 \mathrm{H}, \operatorname{Ar} H), 7.24-7.26(\mathrm{~d}, 2 \mathrm{H}, \operatorname{Ar} H), 5.14(\mathrm{br}, 1 \mathrm{H}$, -NHC(O)O-), 3.27-3.33 (m, 4H, - $\left.\mathrm{NCH}_{2}-\right), 1.58-1.66$ (m, 4H, - $\left.\left(\mathrm{CH}_{2}\right)_{2} \mathrm{CH}_{2}-\right), 1.38-1.46(\mathrm{~m}, 4 \mathrm{H}$, $\left.-\left(\mathrm{CH}_{2}\right)_{2} \mathrm{CH}_{2-}\right) .{ }^{13} \mathrm{C} \mathrm{NMR}\left(\mathrm{CDCl}_{3}, 100 \mathrm{~Hz}\right):{ }^{\mathrm{TM}}=195.8,154.6,153.9,137.8,134.5,132.5,131.8$, 130.1, 128.4, 121.4, 43.0, 41.3, 31.3, 29.9, 26.4, 26.3. MS (ESI): m/z calc. for $\mathrm{C}_{21} \mathrm{H}_{22} \mathrm{~N}_{2} \mathrm{O}_{4},[\mathrm{M}$ $+\mathrm{Na}]^{+}$389.15, found 389.2.

Surface functionalization of CNCs with benzophenone (CNC-g-BP). CNC-g-BP was synthesized according to the protocol of Biyani et al. ${ }^{34}$ In brief, lyophilized cotton CNCs (1.5 g) were dispersed in anhydrous DMF $(595 \mathrm{~mL})$ under nitrogen atmosphere by sonication with a Sonoswiss SW3H ultrasonic unit for $3 \mathrm{~h}$. Then, the flask was covered with aluminum foil and compound $1(3.7 \mathrm{~g}, 10.2 \mathrm{mmol})$ and DBTDL $(220 \mu \mathrm{L})$ were added. The mixture was stirred at 
$100^{\circ} \mathrm{C}$ for $16 \mathrm{~h}$. Then the product was sedimented by centrifugation $(10 \mathrm{~min}$ at $7000 \mathrm{rpm})$, the supernatant was decanted, and the product was washed with DMF $(2 \mathrm{x}, 40 \mathrm{~mL})$, and then THF (3x $40 \mathrm{~mL})$. Finally, CNC-g-BP were transferred into an amber glass vial and dried in high vacuum resulting in a pale yellow powder.

\section{Synthesis of polymethacrylate HNPs via surface-photoinitiated grafting of} polymethacrylates from the CNCs surface and their self-assembly into OCNs materials. In a $25 \mathrm{~mL}$ Schlenk tube equipped with a stirring bar, CNC-g-BP (60 mg) were dispersed in anhydrous DMF ( $8 \mathrm{~mL}$ ) by sonication in a Sonoswiss $\mathrm{SW} 3 \mathrm{H}$ ultrasonic unit for $3 \mathrm{~h}$. Then, the amount of monomer necessary to reach the desired $\mathrm{CNC}$ content (i.e., 4 or $2 \mathrm{~mL}$ of monomer for the synthesis of HNP-10\% or HNP-20\%) were added to the reaction mixture. The mixture was degassed by the freeze-pump-thaw method. In brief, the mixture was frozen in liquid $\mathrm{N}_{2}$, the Schlenk tube was evacuated and the vacuum was maintained for $15 \mathrm{~min}$. Then, the tube was closed and the mixture was allowed to thaw in a water bath at room temperature. This process was repeated until no gas bubbles were observed during thawing, but in any case a minimum of three times. Subsequently, the Schlenk tube was placed between two 6 Watt UV lamps irradiating at $365 \mathrm{~nm}$ to start the surface-initiated polymerization. After $16 \mathrm{~h}$, the reaction mixture clearly separated in two phases (i.e., liquid and highly viscous gel). The reaction mixture was diluted with THF (about $10 \mathrm{~mL}$ ), stirred in a vortex mixer until complete dispersion was achieved, before it was transferred into a falcon tube and the HNPs were separated by ultracentrifugation $(20 \mathrm{~min}$ at $7000 \mathrm{rpm})$. The supernatant was collected, replaced with THF $(20 \mathrm{~mL})$ and the centrifugation/washing process was repeated 3 times. The final white HNP product was dried under high vacuum and the grafted amount of polymer was determined gravimetrically from the amount of CNC-g-BP used and the weight of the dry product isolated (i.e., $823 \mathrm{mg}$ and $332 \mathrm{mg}$ for PMMA-HNP-10\% and PMMA-HNP-20\%, 530 mg and 317 mg for PHMA-HNP-10\% and PHMA-HNP-20\%, respectively. See Table 1 for 
more information). Free polymethacrylates were isolated from the supernatant of the synthesis of the HNPs by concentration under reduced pressure and precipitation in cold methanol. The precipitate was collected by filtration, diluted in a minimum amount of THF, and precipitated again in cold methanol. This dilution/precipitation process was repeated three times. Free PMMA was obtained as white solid $(1.1 \mathrm{~g}, 29 \%$ and $0.5 \mathrm{~g}, 28 \%$ for free polymers isolated from PMMA-HNP-10\% and PMMA-HNP-20\%, respectively), while the free PHMA was obtained as a rubbery transparent solid $(1.9 \mathrm{~g}, 56 \%$ and $0.6 \mathrm{~g}, 35 \%$ for free polymers isolated from PHMA-HNP-10\% and PHMA-HNP-20\%, respectively) (Table 1).

In order to form OCNs, the dried HNPs (about $300 \mathrm{mg}$ ) were redispersed in THF (40 mL) by mechanical stirring, the dispersions were cast into round Teflon Petri dishes with an inner diameter of $77 \mathrm{~mm}$, and dried at $50{ }^{\circ} \mathrm{C}$. After the films had been dried, they were re-processed by compression molding in a Carver CE press at $160{ }^{\circ} \mathrm{C}$ or $130{ }^{\circ} \mathrm{C}$ for $5 \mathrm{~min}$ and $10 \mathrm{sec}$, depending on the grafted polymer (PMMA or PHMA, respectively) to obtain homogeneous films with a thickness of ca. $0.2 \mathrm{~mm}$. To minimize moisture absorption the films were then stored at ambient temperature over drierite in a desiccator, until they were used.

General procedure for the degrafting of polymethacrylate grafts from HNPs. The degrafting of polymethacrylate grafts from HNPs through selective urethane cleavage was performing based on the procedure reported by Greber and Kricheldorf. ${ }^{59}$ In brief, a $5 \mathrm{~mL} 2-$ necked round-bottomed Schlenk flask fitted with a reflux condenser and a stirring bar, the HNPs $(150 \mathrm{mg})$ were dispersed under nitrogen atmosphere in anhydrous THF (4 mL) and vigorous stirring. Then, dry trimethylamine (2.2 equivalent of urethane present in the HNPs) was added and chlorotrimethylsilane (2.2 equivalent of urethane present in the HNPs) was dropwise added. The mixture was placed into an oil bath and stirred at $70{ }^{\circ} \mathrm{C}$ for 4 hours. After this time, the mixture was cooled to room temperature, diluted with $5 \mathrm{~mL}$ of THF, and the 
CNCs were separated by ultracentrifugation. The supernatant containing the cleaved polymer was concentrated under reduced pressure, and then precipitated into $10 \mathrm{~mL}$ of cold methanol. The precipitate was dissolved in THF $(1 \mathrm{~mL})$ and precipitated into $10 \mathrm{~mL}$ of cold methanol. This procedure was repeated 3 times to afford a white precipitate corresponding to the cleaved polymethacrylate (Table S3). 


\section{ASSOCIATED CONTENT}

Supporting information

The supporting information is available free of charge from the XXX website at DOI: XXX.

It includes all Supporting Figures, Tables and calculations employed within this work.

\section{AUTHOR INFORMATION}

\section{Corresponding Author}

*(C.W.) E-mail: christoph.weder@unifr.ch. Telephone: +41 263009465

\section{ORCID}

Sandra Wohlhauser: 0000-0001-5155-1118

Worarin Meesorn: 0000-0003-0554-6910

Lucas Montero de Espinosa: 0000-0003-3616-0287

Justin O. Zoppe: 0000-0002-3599-9227

Christoph Weder: 0000-0001-7183-1790

\section{Notes}

The authors declare no competing financial interest 


\section{ACKNOWLEDGMENTS}

The authors thank Celine Calvino for fruitful discussions about the selective cleavage of urethane groups, Dimitri Vanhecke for his devoted time and helpful advice regarding the acquisition of TEM images, and Gwendoline Delepierre for her help with the AFM. S. Wohlhauser and C. Weder gratefully acknowledge financial support from the US Army Research Office under Grant No. W911NF-15-1-0190 and W911NF-18-1-0287. Dr. W. Meesorn thanks the Swiss Confederation for a doctoral scholarship. Dr. J. O. Zoppe acknowledges the financial support of the Swiss National Science Foundation (SNSF) through Ambizione Grant No. PZ00P2_167900. Dr. L. Montero de Espinosa acknowledges funding through the Ambizione program (Grant No. PZ00P2_154845) of the SNSF. The authors are also thankful for the support of the Adolphe Merkle Foundation. 


\section{REFERENCES}

1. Krishnamoorti, R., Strategies for Dispersing Nanoparticles in Polymers. MRS Bull. 2011, 32 (4), 341-347.

2. Kango, S.; Kalia, S.; Celli, A.; Njuguna, J.; Habibi, Y.; Kumar, R., Surface modification of inorganic nanoparticles for development of organic-inorganic nanocomposites-A review. Prog. Polym. Sci. 2013, 38 (8), 1232-1261.

3. Wohlhauser, S.; Delepierre, G.; Labet, M.; Morandi, G.; Thielemans, W.; Weder, C.; Zoppe, J. O., Grafting Polymers from Cellulose Nanocrystals: Synthesis, Properties, and Applications. Macromolecules 2018, 51 (16), 6157-6189.

4. Tian, C.; Fu, S. Y.; Meng, Q. J.; Lucia, L. A., New insights into the material chemistry of polycaprolactone-grafted cellulose nanofibrils/polyurethane nanocomposites. Cellulose 2016, 23 (4), 2457-2473.

5. Fernandes, N. J.; Koerner, H.; Giannelis, E. P.; Vaia, R. A., Hairy nanoparticle assemblies as one-component functional polymer nanocomposites: opportunities and challenges. MRS Commun. 2013, 3 (1), 13-29.

6. Voudouris, P.; Choi, J.; Gomopoulos, N.; Sainidou, R.; Dong, H.; Matyjaszewski, K.; Bockstaller, M. R.; Fytas, G., Anisotropic Elasticity of Quasi-One-Component Polymer Nanocomposites. ACS Nano 2011, 5 (7), 5746-5754.

7. Chen, G.; Dufresne, A.; Huang, J.; Chang, P. R., A Novel Thermoformable Bionanocomposite Based on Cellulose Nanocrystal-graft-Poly( $\varepsilon$-caprolactone). Macromol. Mater. Eng. 2009, 294 (1), 59-67.

8. Chang, A. C.; Chen, S.; Carter, K. R., Cellulose nanocrystal surface modification via grafting-from sonogashira coupling of poly(ethynylene-fluorene). Cellulose 2018, 25 (10), $5731-5738$.

9. Hansoge, N. K.; Huang, T.; Sinko, R.; Xia, W.; Chen, W.; Keten, S., Materials by Design for Stiff and Tough Hairy Nanoparticle Assemblies. ACS Nano 2018, 12 (8), 7946-7958.

10. Hansoge, N. K.; Keten, S., Effect of Polymer Chemistry on Chain Conformations in Hairy Nanoparticle Assemblies. ACS Macro Lett. 2019, 8 (10), 1209-1215.

11. Habibi, Y.; Lucia, L. A.; Rojas, O. J., Cellulose Nanocrystals: Chemistry, Self-Assembly, and Applications. Chem. Rev. 2010, 110 (6), 3479-3500. 
12. Moon, R. J.; Martini, A.; Nairn, J.; Simonsen, J.; Youngblood, J., Cellulose nanomaterials review: structure, properties and nanocomposites. Chem. Soc. Rev. 2011, 40 (7), 39413994.

13. Kiziltas, E. E.; Kiziltas, A.; Bollin, S. C.; Gardner, D. J., Preparation and characterization of transparent PMMA-cellulose-based nanocomposites. Carbohydr. Polym. 2015, 127, 381-389.

14. Mariano, M.; El Kissi, N.; Dufresne, A., Cellulose nanocrystals and related nanocomposites: Review of some properties and challenges. J. Polym. Sci., Part B: Polym. Phys. 2014, 52 (12), 791-806.

15. Endes, C.; Camarero-Espinosa, S.; Mueller, S.; Foster, E. J.; Petri-Fink, A.; RothenRutishauser, B.; Weder, C.; Clift, M. J. D., A critical review of the current knowledge regarding the biological impact of nanocellulose. J. Nanobiotechnol. 2016, 14 (1), 78.

16. Favier, V.; Canova, G. R.; Cavaille, J.-Y.; Chanzy, H.; Dufresne, A.; Gauthier, C., Nanocomposite materials from latex and cellulose whiskers. Polym. Adv. Technol. 1995, $6(5), 351-355$.

17. Favier, V.; Chanzy, H.; Cavaille, J. Y., Polymer Nanocomposites Reinforced by Cellulose Whiskers. Macromolecules 1995, 28 (18), 6365-6367.

18. Ouali, N.; Cavaille, J. Y.; Perez, J., Elastic, viscoelastic and plastic behavior of multiphase polymer blends. Plast., Rubber Compos. Process. Appl. 1991, 16 (1), 55-60.

19. Takayanagi, M.; Uemura, S.; Minami, S., Application of equivalent model method to dynamic rheo-optical properties of crystalline polymer. J. Polym. Sci., Part C: Polym. Symp. 1964, 5 (1), 113-122.

20. Sapkota, J.; Kumar, S.; Weder, C.; Foster, E. J., Influence of Processing Conditions on Properties of Poly (Vinyl acetate)/Cellulose Nanocrystal Nanocomposites. Macromol. Mater. Eng. 2015, 300 (5), 562-571.

21. Capadona, J. R.; Shanmuganathan, K.; Trittschuh, S.; Seidel, S.; Rowan, S. J.; Weder, C., Polymer Nanocomposites with Nanowhiskers Isolated from Microcrystalline Cellulose. Biomacromolecules 2009, 10 (4), 712-716.

22. Meesorn, W.; Shirole, A.; Vanhecke, D.; de Espinosa, L. M.; Weder, C., A Simple and Versatile Strategy To Improve the Mechanical Properties of Polymer Nanocomposites with Cellulose Nanocrystals. Macromolecules 2017, 50 (6), 2364-2374. 
23. Huang, J.; Chen, Y.; Chang, P. R., Surface Modification of Cellulose Nanocrystals for Nanocomposites. In Surface Modification of Biopolymers, Thakur, V. K.; Singha, A. S., Eds. John Wiley \& Sons, Inc.: 2015; pp 258-290.

24. Kvien, I.; Tanem, B. S.; Oksman, K., Characterization of Cellulose Whiskers and Their Nanocomposites by Atomic Force and Electron Microscopy. Biomacromolecules 2005, 6 (6), 3160-3165.

25. Ljungberg, N.; Bonini, C.; Bortolussi, F.; Boisson, C.; Heux, L.; Cavaillé, J. Y., New Nanocomposite Materials Reinforced with Cellulose Whiskers in Atactic Polypropylene: Effect of Surface and Dispersion Characteristics. Biomacromolecules 2005, 6 (5), 27322739.

26. Ben Azouz, K.; Ramires, E. C.; Van den Fonteyne, W.; El Kissi, N.; Dufresne, A., Simple Method for the Melt Extrusion of a Cellulose Nanocrystal Reinforced Hydrophobic Polymer. ACS Macro Lett. 2012, 1 (1), 236-240.

27. Way, A. E.; Hsu, L.; Shanmuganathan, K.; Weder, C.; Rowan, S. J., pH-Responsive Cellulose Nanocrystal Gels and Nanocomposites. ACS Macro Lett. 2012, 1 (8), 10011006.

28. de Menezes, A. J.; Siqueira, G.; Curvelo, A. A. S.; Dufresne, A., Extrusion and characterization of functionalized cellulose whiskers reinforced polyethylene nanocomposites. Polymer 2009, 50 (19), 4552-4563.

29. Lin, S.; Huang, J.; Chang, P. R.; Wei, S.; Xu, Y.; Zhang, Q., Structure and mechanical properties of new biomass-based nanocomposite: Castor oil-based polyurethane reinforced with acetylated cellulose nanocrystal. Carbohydr. Polym. 2013, 95 (1), 91-99.

30. Ljungberg, N.; Cavaillé, J. Y.; Heux, L., Nanocomposites of isotactic polypropylene reinforced with rod-like cellulose whiskers. Polymer 2006, 47 (18), 6285-6292.

31. Habibi, Y.; Goffin, A.-L.; Schiltz, N.; Duquesne, E.; Dubois, P.; Dufresne, A., Bionanocomposites based on poly( $\varepsilon$-caprolactone)-grafted cellulose nanocrystals by ringopening polymerization. J. Mater. Chem. 2008, 18 (41), 5002-5010.

32. Zoppe, J. O.; Ataman, N. C.; Mocny, P.; Wang, J.; Moraes, J.; Klok, H.-A., SurfaceInitiated Controlled Radical Polymerization: State-of-the-Art, Opportunities, and Challenges in Surface and Interface Engineering with Polymer Brushes. Chem. Rev. 2017, $117(3), 1105-1318$. 
33. Beck, S.; Méthot, M.; Bouchard, J., General procedure for determining cellulose nanocrystal sulfate half-ester content by conductometric titration. Cellulose 2015, 22 (1), 101-116.

34. Biyani, M. V.; Jorfi, M.; Weder, C.; Foster, E. J., Light-stimulated mechanically switchable, photopatternable cellulose nanocomposites. Polym. Chem. 2014, 5 (19), 57165724.

35. Dorman, G.; Prestwich, G. D., Benzophenone Photophores in Biochemistry. Biochemistry 1994, 33 (19), 5661-5673.

36. Majoinen, J.; Walther, A.; McKee, J. R.; Kontturi, E.; Aseyev, V.; Malho, J. M.; Ruokolainen, J.; Ikkala, O., Polyelectrolyte Brushes Grafted from Cellulose Nanocrystals Using Cu-Mediated Surface-Initiated Controlled Radical Polymerization. Biomacromolecules 2011, 12 (8), 2997-3006.

37. Malho, J.-M.; Morits, M.; Löbling, T. I.; Nonappa; Majoinen, J.; Schacher, F. H.; Ikkala, O.; Gröschel, A. H., Rod-Like Nanoparticles with Striped and Helical Topography. ACS Macro Lett. 2016, 5 (10), 1185-1190.

38. Morandi, G.; Thielemans, W., Synthesis of cellulose nanocrystals bearing photocleavable grafts by ATRP. Polym. Chem. 2012, 3 (6), 1402-1407.

39. Zoppe, J. O.; Habibi, Y.; Rojas, O. J.; Venditti, R. A.; Johansson, L.-S.; Efimenko, K.; Österberg, M.; Laine, J., Poly(N-isopropylacrylamide) Brushes Grafted from Cellulose Nanocrystals via Surface-Initiated Single-Electron Transfer Living Radical Polymerization. Biomacromolecules 2010, 11 (10), 2683-2691.

40. Foster, E. J.; Moon, R. J.; Agarwal, U. P.; Bortner, M. J.; Bras, J.; Camarero-Espinosa, S.; Chan, K. J.; Clift, M. J. D.; Cranston, E. D.; Eichhorn, S. J.; Fox, D. M.; Hamad, W. Y.; Heux, L.; Jean, B.; Korey, M.; Nieh, W.; Ong, K. J.; Reid, M. S.; Renneckar, S.; Roberts, R.; Shatkin, J. A.; Simonsen, J.; Stinson-Bagby, K.; Wanasekara, N.; Youngblood, J., Current characterization methods for cellulose nanomaterials. Chem. Soc. Rev. 2018, 47 (8), 2609-2679.

41. Chong, P. Y.; Janicki, S. Z.; Petillo, P. A., Multilevel Selectivity in the Mild and HighYielding Chlorosilane-Induced Cleavage of Carbamates to Isocyanates. J. Org. Chem. 1998, 63 (23), 8515-8521. 
42. Jiao, Y.; Akcora, P., Accelerated brush growth on nanoparticle surfaces by reversible addition-fragmentation chain transfer polymerization. J. Polym. Sci., Part A: Polym. Chem. 2014, 52 (12), 1700-1705.

43. Turgman-Cohen, S.; Genzer, J., Computer Simulation of Controlled Radical Polymerization: Effect of Chain Confinement Due to Initiator Grafting Density and Solvent Quality in “Grafting From” Method. Macromolecules 2010, 43 (22), 9567-9577.

44. Turgman-Cohen, S.; Genzer, J., Simultaneous Bulk- and Surface-Initiated Controlled Radical Polymerization from Planar Substrates. J. Am. Chem. Soc. 2011, 133 (44), $17567-$ 17569.

45. Habibi, Y.; Chanzy, H.; Vignon, M. R., TEMPO-mediated surface oxidation of cellulose whiskers. Cellulose 2006, 13 (6), 679-687.

46. Tsujii, Y.; Ejaz, M.; Sato, K.; Goto, A.; Fukuda, T., Mechanism and Kinetics of RAFTMediated Graft Polymerization of Styrene on a Solid Surface. 1. Experimental Evidence of Surface Radical Migration. Macromolecules 2001, 34 (26), 8872-8878.

47. Natterodt, J. C.; Shirole, A.; Sapkota, J.; Zoppe, J. O.; Weder, C., Polymer nanocomposites with cellulose nanocrystals made by co-precipitation. J. Appl. Polym. Sci. 2018, 135 (24), 45648 .

48. Roman, M.; Winter, W. T., Effect of Sulfate Groups from Sulfuric Acid Hydrolysis on the Thermal Degradation Behavior of Bacterial Cellulose. Biomacromolecules 2004, 5 (5), 1671-1677.

49. Börjesson, M.; Sahlin, K.; Bernin, D.; Westman, G., Increased thermal stability of nanocellulose composites by functionalization of the sulfate groups on cellulose nanocrystals with azetidinium ions. J. Appl. Polym. Sci. 2018, 135 (10), 45963.

50. French, A. D., Idealized powder diffraction patterns for cellulose polymorphs. Cellulose 2014, 21 (2), 885-896.

51. Segal, L.; Creely, J. J.; Martin, A. E.; Conrad, C. M., An Empirical Method for Estimating the Degree of Crystallinity of Native Cellulose Using the X-Ray Diffractometer. Text. Res. J. 1959, 29 (10), 786-794.

52. Poletto, M.; Ornaghi, H. L.; Zattera, A. J., Native Cellulose: Structure, Characterization and Thermal Properties. Materials 2014, 7 (9), 6105-6119. 
53. Chartoff , R. P.; Menczel, J. D.; Dillman, S. H., Dynamic Mechanical Analysis (DMA). In Thermal Analysis of Polymers, Menczel, J. D.; Prime, R. B., Eds. John Wiley \& Sons, Inc.: 2008; pp 387-495.

54. Moy, P.; Weerasooriya, T.; Chen, W.; Hsieh, A., Dynamic Stress-Strain Response and Failure Behavior of PMMA. Proc. ASME Int. Mech. Eng. Congr. Expo., 2003 2003, (37092), 105-109.

55. Cheng, W. M.; Miller, G. A.; Manson, J. A.; Hertzberg, R. W.; Sperling, L. H., Mechanical behaviour of poly(methyl methacrylate). J. Mater. Sci. 1990, 25 (4), 1917-1923.

56. Shen, J.; Chen, C. C.; Sauer, J. A., Effects of sorbed water on properties of low and high molecular weight PMMA: 1. Deformation and fracture behaviour. Polymer 1985, 26 (4), 511-518.

57. Daniel, W. F. M.; Burdyńska, J.; Vatankhah-Varnoosfaderani, M.; Matyjaszewski, K.; Paturej, J.; Rubinstein, M.; Dobrynin, A. V.; Sheiko, S. S., Solvent-free, supersoft and superelastic bottlebrush melts and networks. Nat. Mater. 2015, 15, 183.

58. Lin, N.; Dufresne, A., Surface chemistry, morphological analysis and properties of cellulose nanocrystals with gradiented sulfation degrees. Nanoscale 2014, 6 (10), 53845393.

59. Greber, G.; Kricheldorf, H. R., A New Synthesis of Isocyanates and Isothiocyanates. Angew. Chem., Int. Ed. Engl. 1968, 7 (12), 941-941. 
TOC Graphic

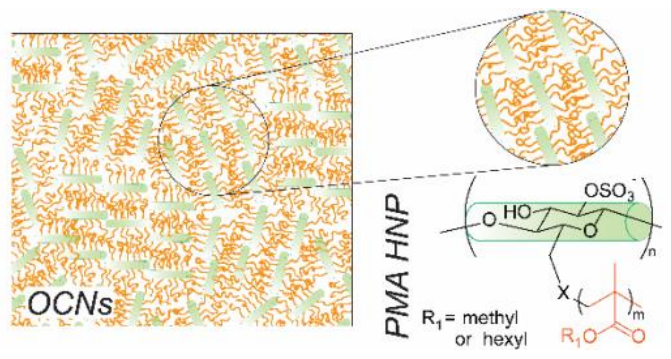

